\title{
Symplectic spatial integration schemes for systems of balance equations
}

\author{
Vu Ngoc Minh Trang ${ }^{a}$, Laurent Lefèvre ${ }^{b}$, Rémy Nouailletas ${ }^{a}$ and Sylvain Brémond ${ }^{a}$ \\ ${ }^{a}$ CEA/DSM/IRFM/STEP, CEA Cadarache, F-13108 Saint-Paul Lez Durance, France \\ ${ }^{b}$ Univ. Grenoble Alpes, LCIS, F-26902, Valence, France
}

\begin{abstract}
A method to generate geometric pseudo-spectral spatial discretization schemes for hyperbolic or parabolic partial differential equations is presented. It applies to the spatial discretization of systems of conservation laws with boundary energy flows and/or distributed source terms. The symplecticity of the proposed spatial discretization schemes is defined with respect to the natural power pairing (form) used to define the port-Hamiltonian formulation for the considered systems of balance equations. The method is applied to the resistive diffusion model, a parabolic equation describing the plasma dynamics in tokamaks. A symplectic Galerkin scheme with Bessel conjugated bases is derived from the usual Galerkin method, using the proposed method. Besides the spectral and energetic properties expected from the symplecticity of the method, it is shown that more accurate approximation of eigenfunctions and reduced numerical oscillations result from this choice of conjugated approximation bases. Finally, the obtained numerical results are validated against experimental data from the tokamak Tore Supra facility.
\end{abstract}

Keywords: symplectic spatial integration, pseudo-spectral methods, balance equations, port-Hamiltonian systems, resistive diffusion equation

\section{Introduction}

Hamiltonian operators are classically used to represent the dynamics of many closed systems of conservation laws. Recently port-Hamiltonian (PH) extensions have been introduced to model open systems with boundary or distributed energy flow [29, 20]. This modelling approach has proven to be fruitful for the modelling, simulation and control of many hyperbolic systems such as transmission line models [13], beam equations [18] or shallow water equations [15]. However, the same approach may as well be applied to 'first principle' parabolic examples such as transport models for adsorption columns [3], fuel cells [11] or diffusion in Ionic Polymer-Metal Composites [23]. Both hyperbolic and parabolic examples make use of a StokesDirac interconnection structure for the realization of the balance equations (e.g. mass, entropy, momentum, etc.).

In the spatial discretization of distributed parameters systems as well as in geometric time integration for ordinary differential equations, pseudo-spectral methods are often chosen because they lead to low order approximate models, with accurate spectral properties (in the linear case, see for instance [10]). Accurate spectral properties and low order models are obviously important features for the design, supervision and control engineering problems. However it is known that key system theoretic properties (both internal or input-output) may be lost in the spatial discretization of distributed parameters systems, when using these pseudo-spectral methods without any additional considerations (as it is also the case for finite-difference schemes). One way of avoiding such problems in the numerical integration of closed Hamiltonian models is to consider geometric methods, i.e. methods which preserve some conserved quantities and/or the geometric interconnection structure of the original model [14]. The latter are usually referred to as symplectic integration methods. The symplecticity is then defined with respect to some Poisson structure. However, these methods usually apply only to the time-integration of closed Hamiltonian systems and not to the spatial discretization of open port-Hamiltonian systems. It must be noticed however that multi-symplectic methods have been developed for the simultaneous space and time integration (i.e. total discretization) of infinite dimensional closed Hamiltonian models [26].

In this paper we define how to systematically build a symplectic spatial discretization scheme for open portHamiltonian systems, starting from any given non symplectic pseudo-spectral discretization method (see for instance [9] for a general presentation of classical pseudospectral methods). Generalizing previous ideas from [6] (mixed finite elements methods) or [22] (mixed orthogonal collocation) we define several approximation bases for the thermodynamical variables, according to their geometric nature (i.e. the degrees of the corresponding differential forms). Then the balance (or conservation) equations and the constitutive (closure) equations are projected into the chosen approximation spaces in order to preserve a power pairing form. The symplecticity of the built mixed pseudo- 
spectral method is thus defined wit respect to this power pairing form, which acts on the distributed and boundary port variables (i.e. the input-output variables).

A second contribution of the paper is related to the choice of approximation spaces. In the proposed construction of mixed symplectic pseudo-spectral spatial discretization methods, the chosen approximation spaces have to be compatible (conjugated) to guarantee the preservation of the power pairing form. Otherwise they could theoretically be chosen quite freely. Classical choices for the approximation spaces are for instance those spanned by Fourier, wavelets or polynomial bases. Not much has been written however about how to choose practically the approximation space among these many possibilities, in the general case. In this work we suggest the use of approximation spaces spanned by conjugated bases of eigenfunctions associated to a simplified problem, this problem being derived by linearization of the original distributed parameters system and spatial uniformization of its parameters. Besides accurate eigenvalues approximations, such choices also provide accurate accurate eigenfunctions approximations. We show on the considered resistive diffusion example how this choice may solve some numerical oscillation problem encountered with distributed actuation or sharp initial conditions.

To illustrate the approach we have chosen to apply these ideas on the example of the port-Hamiltonian formulation for the $1 \mathrm{D}$ resistive diffusion equation [5]. It is a simple plasma control model for the radial diffusion of the poloidal magnetic flux in a tokamak facility. It is a parabolic problem which still may be written in the portHamiltonian formalism using a skew symmetric interconnection structure and toric magnetic coordinates with a homogeneous boundary condition at the center (for symmetry) and a non autonomous (controlled) boundary condition at the outer radius. In this example classical (non symplectic) finite difference or collocation spatial discretization schemes give rise to unwanted numerical oscillating (or even unstable) modes. Simulation results obtained with the proposed symplectic reduction scheme have been validated and compared with experimental data obtained from a discharge of the tokamak Tore Supra discharge (this device is described in [21]).

The paper is organized as follows. In section 2 we recall existing results on the port-Hamiltonian formulation for open distributed parameter systems. In section 2.1 we present the proposed methodology to build mixed symplectic spatial discretization schemes, starting from classical pseudo-spectral methods. In section 4, we derive a mixed Galerkin method for the resistive diffusion problem, using conjugated approximation bases spanned by Bessel's functions. In section 5.1, we analyze numerical results obtained for the resistive diffusion equation with non-uniform resistivity and distributed non inductive current. These numerical results are also compared against against experimental data.

\section{Port-based modelling for systems of balance equa- tions with boundary energy flows}

Quite recently, an intrinsic formulation of port-based models for distributed parameter systems (described by partial differential state space equations) with boundary energy flow have been proposed [29]. It is based on the definition of the state variables as the densities of some thermodynamical extensive variables. The time derivatives of these variables and their conjugated intensive variables ${ }^{1}$ form together the pairs of variables which are used to define a power pairing form and a port-Hamiltonian formulation for systems of conservation laws. Using these variables the usual port Hamiltonian formulation is extended to the infinite-dimensional systems using a canonical geometric interconnection structure called Stokes-Dirac structure [29]. We shall now briefly recall the definitions of these Stokes-Dirac structures and port-Hamiltonian extensions for distributed parameter systems in the 1D case, with a spatial domain $\Omega=[0, L]$.

\subsection{Hamiltonian formulation for systems of conservation laws}

We shall define the conserved quantities as 1-forms on the interval $\Omega=[0, L]$, whose space will be denoted $\Lambda^{1}(\Omega)$. Once a coordinate $x$ (i.e. a measure $d x$ ) has been chosen on the interval $\Omega$, the 1 -form $\alpha \in \Lambda^{1}(\Omega)$ may be written, using this coordinate, $\alpha=\alpha(x) d x$ where $\alpha($.$) denotes$ a smooth function. The state space of a system of two conservation laws is the product space $\Lambda^{1}(\Omega) \times \Lambda^{1}(\Omega)$. The space of 0 -forms, that is smooth functions on the interval $\Omega$, is denoted by $\Lambda^{0}(\Omega)$.

The symbol $\wedge$ will denote the exterior product of $k$ forms and $\mathrm{d}$ the exterior derivative ${ }^{2}$. We shall make use the Hodge star $\star$ operator associated with the measure $d x$ of the real interval $\Omega$. In the coordinate $x$, the Hodge star product of the 1-form $\alpha(x) d x$ is simply the 0 -form: $\alpha(x)$.

Between 0 -form $\Lambda^{0}(\Omega) \ni \beta$ and 1 -form $\Lambda^{1}(\Omega) \ni \alpha$, one may define a bilinear form

$$
<\beta \mid \alpha>:=\int_{\Omega} \beta \wedge \alpha \quad(\in \mathbb{R})
$$

which is simply expressed in coordinates as $\langle\beta \mid \alpha\rangle:=$ $\int_{\Omega} \beta(x) \alpha(x) d x$. The bilinear form (2.1), also referred in this paper as the natural power-pairing, is non-degenerate in the sense that if $\langle\beta \mid \alpha\rangle=0$ for all $\alpha$ (respectively for all

\footnotetext{
${ }^{1}$ Such pairs of conjugated variables are, for instance, the entropy density flow and the temperature in the thermal domain, the momentum density and the velocity in the kinetic domain, the pressure and the volumetric flow in the hydraulic domain, etc.

${ }^{2}$ Actually in the case of a $1 \mathrm{D}$ domain these operations become quite trivial. The wedge product of 0 -forms, i.e. functions, is simply their product and the wedge product of a 0 -form with a 1 -form is again simply the usual product of the 1 -form by the 0 -form. The only non-trivial exterior derivation acts on 0 -forms and is written with the coordinate $x: \mathrm{d} \beta(x)=\frac{\partial \beta}{\partial x}(x) d x$.
} 
$\beta$ ), then $\beta=0$ (respectively $\alpha=0$ ). For real-valued functions $\gamma_{i} \in \Lambda^{0}(\partial \Omega), i=1,2$ associated the spatial domain boundary $\partial \Omega=\{0, L\}$, we define the non-degenerated bilinear form:

$$
\left\langle\gamma_{1}, \gamma_{2}\right\rangle_{\partial}=\int_{\partial \Omega} \gamma_{1} \wedge \gamma_{2}=\gamma_{1}(L) \gamma_{2}(L)-\gamma_{1}(0) \gamma_{2}(0)
$$

Consider an energy density 1-form $\mathcal{H}: \Lambda^{1}(\Omega) \times \Omega \rightarrow$ $\Lambda^{1}(\Omega)$ and denote by $H:=\int_{\Omega} \mathcal{H} \in \mathbb{R}$ the associated Hamiltonian function. Then for any 1-form $\omega \in \Lambda^{1}(\Omega)$ and any variation $\Delta \omega \in \Lambda^{1}(\Omega)$ with compact support strictly included in $\Omega$ and any $\kappa \in \mathbb{R}$, it may be proven that [29] :

$$
\begin{aligned}
H(\omega+\kappa \Delta \omega) & =\int_{\Omega} \mathcal{H}(\omega+\kappa \Delta \omega) \\
& =\int_{\Omega} \mathcal{H}(\omega)+\kappa \int_{\Omega}\left[\frac{\delta H}{\delta \omega} \wedge \Delta \omega\right]+\mathrm{O}\left(\kappa^{2}\right)
\end{aligned}
$$

for a uniquely defined 0 -form which will be denoted $\frac{\delta H}{\delta \omega} \in$ $\Lambda^{0}(\Omega)$ and which is called the variational derivative of $H$ with respect to $\alpha \in \Lambda^{1}(\Omega)$. It should be noticed that when $\mathcal{H}$ only depends on $\omega$ (and not on its derivatives) and when the integration domain is fixed, then $\frac{\delta H}{\delta \omega}=\frac{\partial \mathcal{H}}{\partial \omega}$. We will now define open systems of two conservation laws in canonical interaction.

Definition 2.1. Consider the two conserved quantities as being two 1-forms: $q \in \Lambda^{1}(\Omega)$ and $p \in \Lambda^{1}(\Omega)$. Consider also the system of conservation laws, with flux variables $\beta_{q}$ and $\beta_{p}$ for each conserved quantity, defined by the Hamiltonian density function $\mathcal{H}: \Lambda^{1}(\Omega) \times \Lambda^{1}(\Omega) \times \Omega \rightarrow \Lambda^{1}(\Omega)$ resulting in the total Hamiltonian $H:=\int_{\Omega} \mathcal{H}(q, p) \in \mathbb{R}$. The associated system of two canonically interacting conservation laws is then defined by:

$$
\frac{\partial}{\partial t}\left(\begin{array}{c}
q \\
p
\end{array}\right)+\mathrm{d}\left(\begin{array}{c}
\beta_{q} \\
\beta_{p}
\end{array}\right)=0
$$

and

$$
\left(\begin{array}{l}
\beta_{q} \\
\beta_{p}
\end{array}\right)=\varepsilon\left(\begin{array}{ll}
0 & 1 \\
1 & 0
\end{array}\right)\left(\begin{array}{c}
\frac{\delta H}{\delta q} \\
\frac{\delta H}{\delta p}
\end{array}\right)
$$

where $\varepsilon \in\{-1,+1\}$ depends on the fluxes sign convention on the physical domain.

This system of two conservation laws may be also written as follows:

$$
\frac{\partial}{\partial t}\left(\begin{array}{c}
q \\
p
\end{array}\right)=\varepsilon\left(\begin{array}{ll}
0 & \mathrm{~d} \\
\mathrm{~d} & 0
\end{array}\right)\left(\begin{array}{c}
\frac{\delta H}{\delta q} \\
\frac{\delta H}{\delta p}
\end{array}\right)
$$

that is as an infinite-dimensional Hamiltonian system defined with respect to the matrix differential operator:

$$
\mathcal{J}=\varepsilon\left(\begin{array}{ll}
0 & \mathrm{~d} \\
\mathrm{~d} & 0
\end{array}\right)
$$

and generated by the Hamiltonian function $H[24] .{ }^{3}$

In order to generate a Hamiltonian systems, the matrix differential operator $\mathcal{J}$ defined in (2.4) should satisfy the properties of a Hamiltonian operator, that is it should be skew-symmetric and satisfies the Jacobi identities. Jacobi identities are trivially satisfied for constant differential operators. A short calculus shows that the skew-symmetry holds only for functions with compact support in the spatial domain $\Omega$ or satisfying Dirichlet or Neumann homogeneous boundary conditions.

\subsection{Port Hamiltonian formulation for systems of conser- vation laws with boundary energy flows}

Interested in observation and control problems, one must consider more general (dynamic) boundary conditions where some energy is exchanged through the boundary of the spatial domain. Therefore the matrix differential operator $\mathcal{J}$ must be extended to a Dirac structure, called Stokes-Dirac structure [29, 19, 17]. Dirac structures [7, 8] are a geometric perspective to skew-symmetric tensors, actually corresponding to their graph, which generalize the tensors associated with Poisson brackets or pre-symplectic forms. They have been introduced in classical mechanics to represent systems with constraints. Then they have been used to include input-output port variables in finite dimensional port-Hamiltonian models [28]. Finally an infinite dimensional extension, called Stokes-Dirac structures, has been defined to represent distributed parameters systems of conservation laws with boundary energy flows [29]. Proposition 2.2. [29] Consider the spaces of flows and efforts respectively defined as the product of $k$-forms spaces:

$$
\begin{array}{rlr}
\mathcal{F}=\Lambda^{1}(\Omega) \times \Lambda^{1}(\Omega) \times \Lambda^{0}(\partial \Omega) \ni & \left(f_{p}, f_{q}, f_{b}\right) \\
\mathcal{E}=\Lambda^{0}(\Omega) \times \Lambda^{0}(\Omega) \times \Lambda^{0}(\partial \Omega) \ni & \left(e_{p}, e_{q}, e_{b}\right)
\end{array}
$$

where the subscript $b$ stands for the boundary variables while the subscripts $p$ and $q$ refer to the corresponding conserved quantities or energy domains. Consider the linear subspace $\mathscr{D}$ of the bond space $\mathcal{B}=\mathcal{F} \times \mathcal{E}$ defined as:

$$
\begin{aligned}
\mathscr{D}= & \left\{\left(f_{p}, f_{q}, f_{b}, e_{p}, e_{q}, e_{b}\right) \in \mathcal{F} \times \mathcal{E} \mid\right. \\
& {\left[\begin{array}{c}
f_{p} \\
f_{q}
\end{array}\right]=\varepsilon\left[\begin{array}{cc}
0 & d \\
d & 0
\end{array}\right]\left[\begin{array}{c}
e_{p} \\
e_{q}
\end{array}\right] } \\
& {\left.\left[\begin{array}{c}
f_{b} \\
e_{b}
\end{array}\right]=\left[\begin{array}{cc}
\varepsilon & 0 \\
0 & -1
\end{array}\right]\left[\begin{array}{l}
e_{p \mid \partial \Omega} \\
e_{q \mid \partial \Omega}
\end{array}\right]\right\} }
\end{aligned}
$$

where $\varepsilon \in\{-1,+1\}$ and $\left.\right|_{\partial \Omega}$ denotes restriction to the boundary $\partial \Omega$. Then $\mathscr{D}$ is a Dirac structure with respect to the non degenerated bilinear form:

$$
\begin{aligned}
& \left\langle\left(e_{p}, e_{q}, e_{b}\right) \mid\left(f_{p}, f_{q}, f_{b}\right)\right\rangle \\
= & \int_{\Omega}\left[e_{p} \wedge f_{p}+e_{q} \wedge f_{q}\right]+\left\langle e_{b}, f_{b}\right\rangle_{\partial \Omega}
\end{aligned}
$$

${ }^{3}$ In the coordinates $x$, the Hamiltonian system (2.4) may be written using functions as:

$$
\frac{\partial}{\partial t}\left(\begin{array}{c}
q(x) \\
p(x)
\end{array}\right)=\varepsilon\left(\begin{array}{cc}
0 & \frac{\partial}{\partial x} \\
\frac{\partial}{\partial x} & 0
\end{array}\right)\left(\begin{array}{c}
\frac{\delta H}{\delta q}(x) \\
\frac{\delta H}{\delta p}(x)
\end{array}\right)
$$

In this case the functional spaces may be defined as Hilbert spaces: some more general cases have been studied in [16]. 
For the proof of the condition of co-isotropy: $\mathscr{D}^{\perp} \subset \mathscr{D}$, the reader is referred to [29] and in the $1 \mathrm{D}$ case to [17].

Remark 2.3. A linear subspace $\mathscr{D}$ satisfying only the isotropy condition $\mathscr{D} \subset \mathscr{D}^{\perp}$ (which is therefore not maximal) is called a Tellegen structure. Indeed, it is easily seen that every pair $(f, e)$ in such a $\mathscr{D}$ satisfies

$$
\langle e \mid f\rangle=\int_{\Omega}\left[e_{p} \wedge f_{p}+e_{q} \wedge f_{q}\right]+\left\langle e_{b}, f_{b}\right\rangle_{\partial \Omega}=0
$$

that is instantaneous power conservation in the structure. The Dirac structure (hence the Stokes-Dirac structure) is a generalization of complex interconnection structure from circuit theory. The maximality condition guarantees the uniqueness of this structure associated to the canonical Hamiltonian operator.

As a consequence of proposition 2.2 one may define a Hamiltonian system associated with a Stokes-Dirac structure as follows.

Definition 2.4. The boundary port-Hamiltonian system of two conservation laws with state space $\Lambda^{1}(\Omega) \times \Lambda^{1}(\Omega) \ni$ $(q, p)$ and boundary port variables spaces $\Lambda^{0}(\partial \Omega) \times \Lambda^{0}(\partial \Omega) \ni$ $\left(f_{b}, e_{b}\right)$, is the Hamiltonian system defined with respect to the Stokes-Dirac structure $\mathscr{D}$ given in proposition 2.2 and generated by the Hamiltonian functional $H(q, p)$, as follows:

$$
\left(\left(\left(-\frac{\partial p}{\partial t},-\frac{\partial q}{\partial t}\right), f_{b}\right),\left(\left(\frac{\delta H}{\delta p}, \frac{\delta H}{\delta q}\right), e_{b}\right)\right) \in \mathscr{D}
$$

An appropriate choice of supplementary boundary conditions has to be made to complete this boundary portHamiltonian system in order to obtain a well-posed Cauchy problem (see for instance [16] for a necessary and sufficient characterization of such admissible choices in the linear case). Furthermore, for any solution, the isotropy condition of the Dirac structure implies the balance equation on the Hamiltonian:

$$
\frac{d H}{d t}=\left\langle e_{b}, f_{b}\right\rangle_{\partial}
$$

Remark 2.5. One may also define port variables with support in the spatial domain by considering higher dimensional Hamiltonian operators and the associated Dirac structure $[29,18]$.

\subsection{A parabolic example: the radial resistive diffusion of the magnetic flux in tokamak plasma}

The parabolic resistive diffusion equation describes the diffusion of the poloidal magnetic flux in a plasma fusion facility called Tokamak. In this section we will show, using this example, that Stokes-Dirac structure may be used as well for the formulation of parabolic problems, which may seem quite unusual for the port Hamiltonian approach. In the sequel, the same resistive diffusion equation will be used to illustrate the usefulness of the family of geometric discretization schemes we propose. For this example some other numerical schemes (finite differences, orthogonal collocation, Galerkin), although sometimes used by the plasma control community, are not symplectic (in a sense to be defined later) and therefore lead to known unsatisfactorily dynamical properties of the reduced continuous time model (numerical instabilities and unwanted oscillating modes, for instance).

Using axisymmetry and quasi-static equilibrium (for the pressure) assumptions, it may be proved that magnetic surfaces inside the tokamak chamber are diffeomorphic to nested tori (see Figure 1). In this case, the 3D magnetohydrodynamics equations may be reduced to the $1 \mathrm{D}$ resistive diffusion equation for the poloidal magnetic flux, frequently used to handle the plasma current density profile control problem (see [33] and references therein), which reads:

$$
\frac{\partial \psi}{\partial t}=\eta \frac{1}{C_{3}} \frac{\partial}{\partial x}\left(\frac{1}{\mu} C_{2} \frac{\partial \psi}{\partial x}\right)+\frac{1}{C_{3}}\left(\eta \bar{J}_{n i}\right)
$$

where the normalized "spatial" coordinate $x:=\frac{\rho}{\rho_{\max }} \in$ $\Pi=[0,1]$ denotes the nested surface index (see Figure 1 hereafter). The poloidal magnetic flux, $\psi(t, x)$ is defined as $\partial_{x} \psi=-R_{0} B_{\theta}$ where $R_{0}$ denotes the Tokamak major radius and $B_{\theta}$ the poloidal magnetic flow intensity. $\eta$ is the plasma resistivity, $\mu$ is the void permeability, $C_{2}, C_{3}$ are the coordinate coefficients defined in [5], and $j_{n i}=j_{b s}+j_{e x t}$ is the distributed non inductive current deposit (inside the spatial domain) which includes the bootstrap current $j_{b s}$ described in [32] (a magnetohydrodynamic coupling effect which produces an extra current density) and some external current source terms $j_{\text {ext }}$ controlled through external system heating. Therefore we may consider that $j_{n i}$ is in fact a distributed control or a distributed external source term in the balance equation (2.10).

The boundary conditions will be chosen as:

$$
\left\{\begin{array}{l}
\left.\frac{\partial \psi}{\partial x}\right|_{x=0}=0 \\
\left.\frac{\partial \psi}{\partial t}\right|_{x=1}=V_{\text {loop }}
\end{array}\right.
$$

The first one expresses the smoothness and symmetry with respect to the central circular axis (magnetic axis) of the tokamak torus. The second boundary condition defines a boundary control action. Indeed the central solenoid coil of the tokamak produces an equivalent loop voltage $V_{\text {loop }}$ at the exterior boundary of the plasma (at $x=1$ ). This loop voltage will be considered as one of the controlled inputs (see subsection 3.5).

Using the axisymmetry and quasi-static assumptions, the Maxwell-Ampère and Maxwell-Faraday equations may be written in toric magnetic coordinates in the form of a system where all variables depend only on the magnetic radius $\rho$. Moreover in this reduced 1D system all field variables have only two non trivial components in the poloidal $(\theta)$ and toroidal $(\phi)$ directions since the component in the radial direction vanishes with the quasistatic equilibrium 


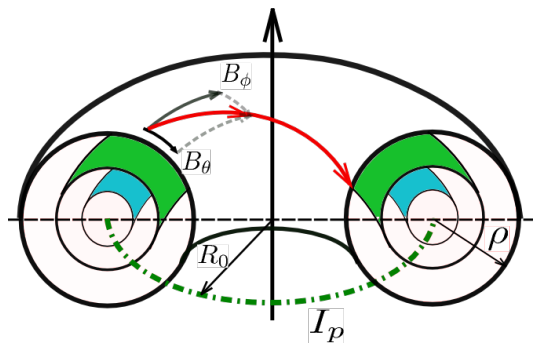

Figure 1: Magnetic toric coordinates. $B_{\theta}$ and $B_{\phi}$ are the two magnetic field components $\left(B_{\rho}=0\right) . \quad R_{0}$ denotes the tokamak major radius and $I_{p}$ the total plasma current. With the axi-symmetry and quasi-static equilibrium assumptions, the state variables only depend on the magnetic surface index $\rho \in\left[0, \rho_{\max }\right]$ and time $t \geq 0$

assumption [5]. Finally, the electromagnetic equations reduce then to the $1 \mathrm{D}$ port-Hamiltonian model [30, 31]:

$$
\left(\begin{array}{c}
f_{\text {el } \phi} \\
f_{m g \theta}
\end{array}\right)=\left(\begin{array}{cc}
0 & -\frac{\partial}{\partial \rho} \\
-\frac{\partial}{\partial \rho} & 0
\end{array}\right)\left(\begin{array}{c}
e_{e l \phi} \\
e_{m g \theta}
\end{array}\right)+\left(\begin{array}{l}
1 \\
0
\end{array}\right) f_{d \phi}
$$

In (2.12) we used the notations $f$ and $e$ for the flow and effort conjugated variables (i.e. their product is a power) with the subscripts $e l, m g$ an $d$ for the electric, magnetic and dissipation variables, where $f_{\text {el } \phi}=-\partial_{t}\left(\rho D_{\phi}\right), f_{m g \theta}=$ $-\partial_{t}\left(R_{0} B_{\theta}\right), e_{e l \phi}=R_{0} E_{\phi}, e_{m g \theta}=\rho H_{\theta}$ and $f_{d \phi}=\rho J_{\phi}$. We adopted quite usual notaions where $B, D$ and $J$ denote respectively the magnetic flux, the electric flux and the current density while $E$ and $H$ denote respectively the electric and magnetic fields intensities

In the resistive diffusion model, the displacement current $f_{\text {el } \phi}$ is considered as negligible when compared to the inductive current. This assumption will result in a system of only one conservation law (related to the magnetic field intensity storage) which can be written as an algebraicdifferential $^{4}$ system using the same 1D Stokes-Dirac structure as previously defined:

$$
\left(\begin{array}{c}
0 \\
f_{m g \theta}
\end{array}\right)=\left(\begin{array}{cc}
0 & -\frac{\partial}{\partial \rho} \\
-\frac{\partial}{\partial \rho} & 0
\end{array}\right)\left(\begin{array}{c}
e_{e l \phi} \\
e_{m g \theta}
\end{array}\right)+\left(\begin{array}{l}
1 \\
0
\end{array}\right) f_{d \phi}
$$

There is no flows source term at $x=0$ (center of the tokamak) and there is a control action at $x=1$ where the loop voltage $V_{\text {loop }}$ is imposed to the plasma by the external coils, that is:

$$
\left\{\begin{array}{c}
e_{e l \phi}(1, t)=-\left.\partial_{t} \psi\right|_{x=1}=-V_{\text {loop }}(t) \\
e_{m g \theta}(0, t)=\left.\frac{x a}{\mu R_{0}} B_{\theta}\right|_{x=0}=0
\end{array}\right.
$$

\footnotetext{
${ }^{4}$ In this context, "algebraic-differential" is an improper term since the dissipation equation also makes use of an exterior derivatives. It only means that the dissipation equation contains no time derivative.
}

The magnetic constitutive equation and the Ohm's law are used as closure equations:

$$
\left\{\begin{array}{ccc}
e_{e l \phi}=\eta \frac{R_{0}}{\rho} J_{\Omega} & \text { Ohm's law } \\
e_{m g \theta}=\frac{\rho}{\mu R_{0}} B_{\theta} & \text { magnetic constitutive equation }
\end{array}\right.
$$

with the ohmic current being defined as:

$$
J_{\Omega}=f_{d \phi}-J_{n i} \text { and } J_{n i}=\rho j_{n i}
$$

The port-Hamiltonian boundary control model model equivalent to the usual resistive diffusion equation is then defined using the balance equations (2.13) together with the closure equations (2.15) and the non homogeneous boundary conditions (2.14). This example will be used throughout the paper to illustrate the proposed geometric discretization scheme.

Remark 2.6. Note that the proposed model (2.13) for the plasma resistive diffusion makes use of the usual StokeDirac skew-symmetric structure. This seemingly unusual feature is in fact a characteristic of all diffusion-like models and relies on a fundamental assumption from thermodynamics: flows are generated by generalized forces which may be written as gradients of generalized potentials. Once this assumption holds, then a similar transformation into port-Hamiltonian model may be successfully operated with the two formally adjoint operator div (used for the conservation law) and grad used for the flux law. This approach has already been applied for the modelling of transport phenomena [3] and leads to very nice properties of reduction schemes for parabolic equations using spatially symplectic reduction schemes [4].

\section{Discrete Stokes-Dirac structures and structure preserving spatial discretization}

In order to get a spatial finite-dimensional approximation for the original distributed parameters system written in port Hamiltonian form, we will define different specific approximation spaces for the flows and efforts variables which are respectively differentials forms of order 0 and 1 .

This allows to guarantee that the reduced (finite dimensional approximation) variables also satisfy the StokesDirac interconnection relations by performing exact spatial differentiation in the conjugated approximation spaces.

Therefore, the original (spectral, Galerkin, collocation or any other pseudo-spectral) approximation scheme will be transformed into a symplectic one in the sense that the bilinear power pairing form used to define the Stokes-Dirac structure is preserved. Symplecticity with respect to this form ensures that balance equations (and thus conservativeness, dissipativeness and qualitative properties of the spectrum, in the linear case) are satisfied by the finite dimensional approximation even in the case of systems with 
boundary energy flow ${ }^{5}$ which is not the case with classical symplecticity (used for instance for closed Hamiltonian systems).

In this section the presented methodology will be applied on the example of the Tokamak system (2.12) to illustrate how the closure relations may be projected into the approximation bases once they have been chosen (in order to preserve the power bilinear form). Except for the projections of these closure relations, the results of the section are general.

\subsection{Approximation bases for flows and efforts}

The flows $\left(f_{e l}, f_{m g}, f_{d}\right)$ and efforts $\left(e_{e l}, e_{m g}, e_{d}\right)$ are approximated in the 1D $\Pi$ domain using a classical expansion:

$$
\begin{aligned}
& f(t, x)=\sum_{k=1}^{N-1}(\mathbf{f}(t))_{k} w_{k}^{f}(x) \\
& e(t, x)=\sum_{i=1}^{N}(\mathbf{e}(t))_{i} w_{i}^{e}(x)
\end{aligned}
$$

in which $\mathbf{f}(t) \in \mathbb{R}^{N-1}, \mathbf{e}(t) \in \mathbb{R}^{N}$ are the flow and effort time dependent coefficients; $w_{k}^{f}(x)$ and $w_{i}^{e}(x)$ are the base functions satisfying the exact differentiation condition:

$$
\begin{cases}\overline{\mathcal{E}} & =\operatorname{span}\left(w_{i}^{e}(x)\right) \\ \overline{\mathcal{F}} & =\operatorname{span}\left(w_{k}^{f}(x)\right) \\ \mathrm{d}(\overline{\mathcal{E}}) & =\overline{\mathcal{F}}\end{cases}
$$

where $\mathrm{d}$ is the spatial exterior derivative for the corresponding differential form (here it is simply the usual derivative with respect to the reduced spatial coordinate $x$ and applies to effort variables which are 0-forms or functions). From (3.1) and (3.2), we get:

$$
\sum_{k=1}^{N-1} w_{k}^{f}(x) \mathbf{f}_{k}(t)=\sum_{i=1}^{N} \partial_{x}\left(w_{i}^{e}(x)\right) \mathbf{e}_{i}(t)
$$

Since the approximation bases have been chosen to satisfy the exact differentiation condition, the quantities $\partial_{x} w_{i}^{e}(x)$ may be expanded in the flow basis. This results in a finitedimensional derivative operator represented by the differentiation matrix $D \in \mathbb{R}^{(N-1) \times N}$ such that:

$$
\mathbf{f}(t)=D \mathbf{e}(t)
$$

\subsection{Bilinear power product and discrete Stokes' theorem}

On the 1D domain $\Pi$ with boundary $\partial \Pi$, canonical Stokes-Dirac structure may be defined as self-orthogonal subspace $\mathscr{D}$ of the bond space $\mathcal{B}=\mathcal{F} \times \mathcal{E}$ with respect to the inner product defined by symmetrization of the non degenerated bilinear form (or power pairing, see proposition 2.2):

$$
\begin{array}{rlrl}
\langle. \mid .\rangle: \quad & \mathcal{F} \times \mathcal{E} & \longrightarrow & \mathbb{R} \\
(f, e) & \longmapsto & \langle e \mid f\rangle
\end{array}
$$

\footnotetext{
${ }^{5}$ This case is fundamental for control engineering systems where interactions with the environment through measurements and actuations are necessary
}

with

$$
\begin{aligned}
\langle e \mid f\rangle & =\left\langle\left(e_{p}, e_{q}, e^{\partial}\right) \mid\left(f_{p}, f_{q}, f^{\partial}\right)\right\rangle \\
& :=\int_{\Pi}\left[e_{p} \wedge f_{p}+e_{q} \wedge f_{q}\right]-\int_{\partial \Pi} e^{\partial} \wedge f^{\partial}
\end{aligned}
$$

This inner product is then defined as the symmetric positive definite bilinear form:

$$
\begin{array}{llll}
\langle\langle., .\rangle\rangle: & \mathcal{B} \times \mathcal{B} & \mathbb{R} \\
& \left(\left(f_{1}, e_{1}\right),\left(f_{2}, e_{2}\right)\right) & \longmapsto & \left\langle\left\langle\left(f_{1}, e_{1}\right),\left(f_{2}, e_{2}\right)\right\rangle\right\rangle \\
& & :=\left\langle e_{1} \mid f_{2}\right\rangle+\left\langle e_{2} \mid f_{1}\right\rangle
\end{array}
$$

It has been noted in section 2.3 that every pairs $(f, e)$ belonging to the Stokes-Dirac structure (thus satisfying the balance equations) also satisfy $\langle e \mid f\rangle=0$. As a consequence, balance equations (and conservations laws) will be satisfied also in the finite dimensional approximation spaces if the bilinear form (3.6) is preserved by the discretization. If this happens, we will call the reduction scheme symplectic with respect to the power pairing by analogy with symplecticity of time integrators for Hamiltonian (finite dimensional) systems. The relation $\langle e \mid f\rangle=$ 0 (and the resulting symplecticity) will be satisfied for pairs of approximated flow and effort variables defined in the previous section provided that the boundary variables $\left(e^{\partial}, f^{\partial}\right)$ are correctly defined. We will obtain this result for the considered schemes because they perform exact spatial derivation in the chosen compatible conjugated approximation spaces.

In our example of Tokamak electromagnetic system, we can identify:

$$
\begin{aligned}
(e, f) & =\left(\left(e_{p}, e_{q}, e^{\partial}\right),\left(f_{p}, f_{q}, f^{\partial}\right)\right) \\
& :=\left(\left(e_{e l}, e_{m g}, e_{e l}^{\partial}\right),\left(f_{e l}, f_{m g}, e_{m g}^{\partial}\right)\right)
\end{aligned}
$$

Note that each of the efforts and flows have both poloidal $\theta$ and toroidal $\phi$ components.

To perform an exact integration of the power pairing integral (and thus preservation of the inner product in the Bond space) we define the (integral) matrix $M \in$ $\mathbb{R}^{N \times(N-1)}$ as:

$$
M_{i k}=\int_{\Pi} w_{i}^{e}(x) w_{k}^{f}(x) \mathrm{d} x
$$

and the (trace) matrices $T_{k} \in \mathbb{R}^{N \times N}$ as:

$$
\left(T_{k}\right)_{i j}=w_{i}^{e}(x=k) w_{j}^{e}(x=k) \text { where } k \in\{0,1\}
$$

We replace the effort and flow variables by their approximation (3.1) in the chosen conjugated approximation spaces and force the reduced variables to satisfy the Stokes-Dirac equations (2.6) (by exact spatial differentiation). Then we may apply the Stokes theorem (on the power pairing integral) to prove that the bilinear power product computed with the reduced variables coordinates is indeed zero. Therefore, one gets for every pairs of reduced variables $\left(e_{1}, f_{1}\right),\left(e_{2}, f_{2}\right)$ in the Stokes-Dirac struc- 
ture:

$$
\begin{gathered}
\mathbf{e}_{p}^{1 T}\left(\begin{array}{cc}
0 & M D+D^{T} M^{T}-T_{1}+T_{0} \\
-D^{T} M^{T}-M D+T_{1}-T_{0} & 0
\end{array}\right) \mathbf{e}_{q}^{2} \\
-\mathbf{e}_{q}^{1 T}\left(\begin{array}{cc}
0 & M D+D^{T} M^{T}-T_{1}+T_{0} \\
-D^{T} M^{T}-M D+T_{1}-T_{0} & =\mathbf{e}_{p}^{2} \\
=0 & 0
\end{array}\right.
\end{gathered}
$$

where $\mathbf{e}_{p}=\left(\mathbf{e}_{p \theta}, \mathbf{e}_{p \phi}\right)$ and $\mathbf{e}_{q}=\left(\mathbf{e}_{q \theta}, \mathbf{e}_{q \phi}\right)$ are any real vector valued coordinates of the poloidal and toroidal components of the effort variables in the finite dimensional approximation spaces. We have proven that, for any conjugated approximation spaces for reduced efforts and flows which satisfy the compatibility condition (3.2):

$$
M D+D^{T} M^{T}-T_{1}+T_{0}=0
$$

Theorem 3.1 (Discrete Stokes theorem). Let $w_{k}^{f}(x)(k \in$ $\{1, \ldots, N-1\})$ and $w_{i}^{e}(x)(k \in\{1, \ldots, N\})$ denote conjugated approximation bases functions for flow and effort variables which satsify the compatibility condition (3.2). Let $D$ denote the (exterior) differentation matrix as defined in (3.4), $M$ the integration matrix as defined in (3.9) and $T_{k}(k \in\{0,1\})$ the trace matrix as defined in (3.10), Then

$$
M D+D^{T} M^{T}-T_{1}+T_{0}=0
$$

Remark 3.2. Note that for homogeneous boundary conditions, the efforts are zero at the boundary and therefore the trace matrices may be selected as $T_{1}=T_{0}=0$. In this case, there's no energy exchange through the boundary and the discrete Stokes theorem reduces to $M D+$ $D^{T} M^{T}=0$. In this case the matrix $M D$ is skew-symmetric and defines a Poisson tensor. The condition (3.12) may thus be viewed as the extension of a skew-symmetry property characterizing finite dimensional reduced Dirac structures.

Remark 3.3. The discrete Stokes theorem (3.12) is the key property of the proposed family of pseudo-spectral reduction schemes that we propose in this paper. It ensures the symplecticity of every scheme in this family with respect to the "natural" power pairing of the considered system (e.g. (3.6) in our running plasma example). For the considered family of pseudo-spectral schemes, another interpretation may be provided. Indeed in the selected "effort" basis, one may assert that the infinite dimensional spatial derivation operator reduces to the finite dimensional realization (3.4). On the other hand, the exact integration formula and the resulting discrete Stokes theorem ensure that the residual is zero in the approximation space (spanned by the same "effort" basis). Therefore, the proposed mixed pseudo-spectral methods may be also viewed as symplectic Galerkin-type methods realizing the projection of the actual dynamics in the approximation space spanned by the effort basis.

\subsection{Spatial discretization of Stokes-Dirac structure and reduced finite dimensional Dirac structure}

In the case of the resistive diffusion model (2.13), it is possible to study only the poloidal subsystem describing the dynamics of the variables ${ }^{6}$ :

$$
\begin{aligned}
(e, f) & =\left(\left(e_{p}, e_{q}, e^{\partial}\right),\left(f_{p}, f_{q}, f^{\partial}\right)\right) \\
& :=\left(\left(e_{e l \phi}, e_{m g \theta}, e_{e l \phi}^{\partial}\right),\left(f_{e l \phi}, f_{m g \theta}, e_{m g \theta}^{\partial}\right)\right)
\end{aligned}
$$

Indeed the poloidal and toroidal subsystems are totally decoupled and the finite dimensional approximation of the poloidal subsystem may be written independently from the toroidal variables in the form:

$$
\left\{\begin{array}{l}
\left(\begin{array}{c}
\mathbf{f}_{e l \phi} \\
\mathbf{f}_{m g \theta}
\end{array}\right)=\left(\begin{array}{cc}
0 & -D \\
-D & 0
\end{array}\right)\left(\begin{array}{l}
\mathbf{e}_{e l \phi} \\
\mathbf{e}_{m g \theta}
\end{array}\right)+\left(\begin{array}{l}
1 \\
0
\end{array}\right) \mathbf{f}_{d \phi} \\
\left(\begin{array}{c}
f_{1 \phi} \\
f_{0 \phi} \\
e_{1 \theta} \\
e_{0 \theta}
\end{array}\right)=\left(\begin{array}{cc}
w^{e}(1) & 0 \\
w^{e}(0) & 0 \\
0 & w^{e}(1) \\
0 & w^{e}(0)
\end{array}\right)\left(\begin{array}{c}
\mathbf{e}_{e l \phi} \\
\mathbf{e}_{m g \theta}
\end{array}\right)
\end{array}\right.
$$

Therefore, for the sake of simplicity, we will consider throughout the rest of this paper only the poloidal subsystem (3.13). Since the flow variables are obtained from the effort variables through a spatial derivation, the two approximation spaces do not have the same dimension and system (3.13) is not in minimal form. This minimal form is of prime importance because it is required to provide a "causality free" reduced Dirac structure and finite dimensional model, that is a model which may be connected to any other compatible port-controlled Hamiltonian system where any of the flow or effort variables may be freely chosen as an input. To obtain such a minimal form for the system system (3.13) - corresponding to an invertible representation of the reduced Dirac structure - it will be necessary to project the reduced effort variables with a projector which cancels the kernel of the exterior derivative (in the space of reduced effort coordinates) but without affecting the power pairing value and the resulting symplecticity of the reduction scheme. Such a projector is built hereafter. It is based on the exact integration of the power product and is used to pull the system back to a square invertible one.

Using the $\left\{w_{i}^{e}, w_{k}^{f}\right\}$ bases integration matrix $M$ define in (3.9), the power balance equation for the poloidal electromagnetic energy may be exactly evaluated (in the chosen approximation spaces) with the expression:

$$
\begin{aligned}
\frac{\partial \mathbb{H}_{p o l}}{\partial t} & =\int_{\Pi} e_{e l \phi} \wedge f_{e l \phi}+e_{m g \theta} \wedge f_{m g \theta} \\
& =\quad \mathbf{e}_{e l \phi}^{T} M \mathbf{f}_{e l \phi}+\mathbf{e}_{m g \theta}^{T} M \mathbf{f}_{m g \theta}
\end{aligned}
$$

where $\mathbb{H}_{\text {pol }}$ stands for the total "poloidal" Hamiltonian, that is the part of the electromagnetic energy stored in the poloidal subsystem. As mentioned above, the bilinear power product in (3.14) is degenerated and admits the kernel $\operatorname{ker}\left(M^{T}\right)$. Therefore a projection:

$$
\tilde{\mathbf{e}}=M^{T} \mathbf{e}
$$

\footnotetext{
${ }^{6}$ Remember that the resistive diffusion equation is obtained by neglecting the displacement current $f_{p}=f_{e l \phi}=0$. The parabolic resistive diffusion model results from the magnetic balance equation and the closure (diffusion) constitutive equation relating the diffusive flow $-\mathrm{d}\left(e_{m g \theta}\right)$ and the magnetic field intensity $e_{m g \theta}$
} 
allows to obtain an invertible system in the new Bond space of reduced effort and flow variables $\mathbb{R}^{N-1} \times \mathbb{R}^{N-1} \ni$ $(\tilde{\mathbf{e}}, \mathbf{f})$. The degenerated bilinear product (3.14) becomes non degenerated in this new Bond space and the power balance becomes

$$
\frac{\partial \mathbb{H}}{\partial t}=\int_{\Pi} e \wedge f=\mathbf{e}^{T} M \mathbf{f}=\tilde{\mathbf{e}}^{T} \mathbf{f}
$$

The symmetrization of this power pairing results in a bilinear form in the Bond space which is symmetric positive definite, that is defines an inner product. It may be observed that the reduced (invertible) Dirac structure corresponding to this inner product may be written using its image representation, in the form

$$
\begin{aligned}
\left(\begin{array}{c}
\mathbf{f}_{\text {el }} \\
\mathbf{f}_{m g \theta} \\
f_{\partial \phi}^{1} \\
f_{\partial \phi}^{0}
\end{array}\right) & =\underbrace{\left(\begin{array}{cc}
0 & -D \\
-D & 0 \\
w^{e}(1) & 0 \\
w^{e}(0) & 0
\end{array}\right)}_{E^{T}}\left(\begin{array}{c}
\mathbf{e}^{q} \\
\mathbf{e}^{p}
\end{array}\right) \\
\left(\begin{array}{c}
\tilde{\mathbf{e}}_{\mathbf{e l} \phi} \\
\tilde{\mathbf{e}}_{\mathbf{m g} \theta} \\
e_{\partial \theta}^{1} \\
e_{\partial \theta}^{0}
\end{array}\right) & =\underbrace{\left(\begin{array}{cc}
M^{T} & 0 \\
0 & M^{T} \\
0 & w^{e}(1) \\
0 & w^{e}(0)
\end{array}\right)}_{F^{T}}\left(\begin{array}{c}
\mathbf{e}_{\mathbf{e l} \phi} \\
\mathbf{e}_{\mathbf{m g} \theta}
\end{array}\right)
\end{aligned}
$$

The discrete Stokes theorem proven here above allows to prove that $[E: F]$ is full rank and that $E F^{T}+F E^{T}=0$. These are necessary and sufficient conditions to prove that (3.17) indeed defines a well-posed Dirac structure [8]. The relations (3.17) defining the Dirac structure may also be written in explicit ("input-output") form:

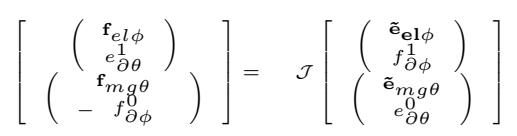

$\mathcal{J}=\left[\begin{array}{cc}0 & \left(\begin{array}{c}-D \\ w^{e}(1)\end{array}\right)\left(\begin{array}{c}M^{T} \\ w^{e}(0)\end{array}\right)^{-1} \\ 0 & \end{array}\right]$ is a skew matrix as $\mathcal{J}+\mathcal{J}^{T}=0$ thanks to (3.12).

\subsection{Constitutive relations}

Relations between efforts and flows variables coming from the balance equations and realized with the Dirac interconnection structure have to be completed with closure relations. These come from the constitutive equations considered in each specific example. Usually they are storage and dissipation phenomenological laws. They may be projected in the finite-dimensional approximation spaces which have been previously chosen to guarantee symplecticity of the spatial reduction scheme. This means that the energy stored and dissipated in the actual dynamics will be equally stored and dissipated in the reduced dynamics although the value of these energies will only be approximated ones. Energy storage and dissipation reductions are illustrated hereafter on the plasma model example.

\subsubsection{Energy storage element}

From (3.14), the poloidal magnetic power may be written:

$$
\frac{d \mathbb{H}_{m g}}{d t}=\int_{\Pi} e_{m g \theta} \wedge f_{m g \theta}=\tilde{\mathbf{e}}_{m g \theta}^{T} \mathbf{f}_{m g \theta}
$$

On the other hand, the quadratic form for the magnetic energy results in a linear constitutive equation with the magnetic effort variable defined as: $e_{m g \theta}=\frac{a x}{\mu R_{0}} B_{\theta}$. Hence the poloidal magnetic power may be projected in the chosen flow basis according to:

$$
\begin{aligned}
& \frac{d \mathbb{H}_{m g \theta}}{d t}=\int_{\Pi} e_{m g \theta} \wedge f_{m g \theta}=\int_{\Pi} \frac{C_{2}(x)}{\mu} B_{\theta} \wedge \frac{\partial B_{\theta}}{\partial t} \\
& \simeq \quad \mathbf{b}^{T} G \mathbf{f}_{m g \theta} \\
& \text { with } G_{i j}=\int_{\Pi} \frac{C_{2}(x)}{\mu} w_{i}^{f}(x) w_{j}^{f}(x) \quad G=G^{T}>0 \\
& \text { and } B_{\theta}(t, x)=\sum_{k=1}^{N-1}(\mathbf{b}(t))_{k} w_{k}^{f}(x)
\end{aligned}
$$

This results in the finite dimensional magnetic constitutive equation:

$$
\tilde{\mathbf{e}}_{m g \theta}=G \mathbf{b}
$$

\subsubsection{Energy dissipative element}

The dissipated poloidal power is defined as:

$$
\mathbb{P}_{d \phi}=\int_{\Pi} e_{d \phi} \wedge f_{d \phi}=\tilde{\mathbf{e}}_{e l \phi}^{T} \mathbf{f}_{d \phi}
$$

using for instance Ohm's law for the electrical diffusion: $e_{d \phi}=\frac{\eta}{C_{3}(x)} J_{\Omega}=\frac{\eta}{C_{3}(x)}\left(f_{d \phi}-J_{n i}\right)$, this poloidal dissipation may be approximated in the same flow basis:

$$
\begin{aligned}
\mathbb{P}_{d \phi} & =\int_{\Pi} e_{d \phi} \wedge f_{d \phi}=\int_{\Pi} \frac{\eta}{C_{3}(x)}\left(f_{d \phi}-J_{n i}\right) \wedge f_{d \phi} \\
& \simeq \quad\left(\mathbf{f}_{d \phi}-\mathbf{J}_{\mathbf{n i}}\right)^{T} R \mathbf{f}_{d \phi}
\end{aligned}
$$

with the dissipation matrix $R$ is defined as

$$
R_{i j}=\int_{\Pi} \frac{\eta}{C_{3}(x)} w_{i}^{f}(x) w_{j}^{f}(x) \quad R=R^{T}>0
$$

Hence the dissipative constitutive equation may be written:

$$
\tilde{\mathbf{e}}_{e l \phi}=R\left(\mathbf{f}_{d \phi}-\mathbf{J}_{\mathbf{n i}}\right)
$$

\subsection{Boundary conditions}

When dealing with boundary control systems, two kinds of boundary conditions have to be distinguished: homogeneous and time varying boundary conditions. Most often homogeneous boundary conditions are used for symmetry considerations or permanent physical interconnection 
(contact condition, isolated systems, etc.). On the contrary, time varying boundary conditions are used to represent external control actions on the system. This boundary control actions correspond to a boundary energy flow and are realized by forcing time varying efforts and/or flows values at the system boundary.

In our approach, the homogeneous boundary conditions (with no power exchange with the environment) will be integrated in the approximation bases choice: all homogeneous boundary conditions on the effort or flow values (and their spatial derivatives) will be satisfied by all functions in the corresponding approximation bases. This common choice very often allows to get better results in terms of accuracy, minimizing of the boundary effects introduced by the boundary conditions. Besides, it leads to some symplectic reduction schemes for homogeneous problems as it may be seen from results in the next section or in $[22]$.

On the contrary, non homogeneous boundary conditions - since they are time varying - have to be considered as additional relations in the finite dimensional state space model. Usually they break symmetries in this model and lead to a lack of symplecticity in the considered spatial discretization scheme [22]. In our approach these boundary conditions are time varying values either for the effort or flow boundary values. The proposed family of schemes remain symplectic because symplecticity is defined with respect to a bilinear form which "embed" the boundary variables (this is precisely the aim of the extension from the Poisson to the Dirac structure).

These ideas are illustrated hereafter on the plasma example, both for homogeneous boundary conditions and for boundary control actions.

\subsubsection{Homogeneous boundary conditions}

We consider that there is no flow source inside the domain at $x=0$ (the central magnetic axis inside the toroidal plasma chamber). Therefore the following symmetry boundary conditions apply

$$
f_{d \phi}(0)=f_{m g \theta}(0)=0
$$

since $f_{d \phi}=\frac{1}{C_{3}} J_{\phi}$ and $f_{m g \theta}=\partial_{t} B_{\theta}=-\partial_{t} \frac{\partial \psi}{\partial x}$ with $\left.\frac{\partial \psi}{\partial x}\right|_{x=0}=0$. On another hand, the constitutive relation $e_{m g \theta}=\frac{C_{2}}{\mu} B_{\theta}$ and $C_{2}(0)=0$ leads to:

$$
e_{0 \theta}=e_{m g \theta}(0)=0
$$

\subsubsection{Non-homogeneous boundary conditions}

The loop voltage will be considered as the controlled boundary input. Therefore we will consider non homogeneous boundary conditions at the boundary $x=1$ :

$$
f_{1 \phi}=e_{e l \phi}(1)=-\left.\partial_{t} \psi\right|_{1}=-V_{\text {loop }}(t)
$$

Taking into account the quasi-static assumption $f_{\text {el } \phi}=$ 0 , the interconnection equations (3.18), together with the energy storage constitutive equation (3.23), the energy dissipation constitutive equation (3.27) and the boundary conditions here above, we may the complete reduced state space in the following $\mathrm{PCH}$ form:

$$
\left.\left(\begin{array}{c}
\left(\begin{array}{c}
-\mathbf{f}_{d \phi} \\
e_{1 \theta}
\end{array}\right) \\
-\dot{\mathbf{b}} \\
-f_{0 \phi}
\end{array}\right)\right)=\mathcal{J}\left(\begin{array}{c}
R\left(\mathbf{f}_{d \phi}-\mathbf{J}_{\mathbf{n i} \phi}\right) \\
-V_{l o o p} \\
\left(\begin{array}{c}
G \mathbf{b} \\
0
\end{array}\right)
\end{array}\right)
$$

The model (3.31) will be used in the sequel to compute the approximated eigenvalues of the finite dimensional approximations obtained by the geometric discretization schemes proposed in this paper.

\section{A mixed symplectic Galerkin scheme}

In this section we investigate the problem of the selection of conjugated approximation bases for the efforts and flows. This problem of choosing the approximation space is common to any pseudo-spectral method (whatever it is symplectic or not). Its solution is usually dependent both of the the properties of the actual system and the expected properties for the reduced one. It is quite usual in control problems to choose a polynomial approximation space. Such a space could be generated by any polynomial basis. For instance, using Lagrange interpolation polynomials will lead to define the approximation coordinates simply as the values of the variables spatial profiles at the collocation points. It is quite usual to choose collocation points as zeros of orthogonal polynomials since this choice maximizes the order of accuracy of the corresponding interpolation methods. With such a choice, the resulting symplectic collocation scheme which is derived, using the method proposed in this paper, should produce a finite dimensional $\mathrm{PCH}$ model easy to manage for subsequent control problems since it is low order, its state space variables have a physical meaning and its spectral properties should be close to the actual ones (this is a common feature from pseudo-spectral schemes). It is indeed the case. This resulting symplectic collocation spatial discretization method is presented with some details in Appendix B. However, as it is shown in Appendix B, this choice do not allow - in the considered plasma control problem - to handle the distributed control action (non inductive current deposit). This will be explained by a poor approximation of the eigenfunctions of the considered operators although the approximation of its eigenvalues is indeed quite accurate (see Appendix $\mathrm{C}$ for details).

Therefore we will choose another approximation basis for the efforts. It is suggested by the observation that, with an uniform resistivity $\eta$, the eigenvalues problem for the plasma resistive diffusion equation gives rise to a Bessel equation. Hence eigenfunctions for this simplified uniform problem are Bessel functions and the eigenvalues can be explicitly evaluated from the zeros of Bessel functions (details of these computations are in Appendix A). We will 
thus select the effort approximation basis as spanned by these Bessel functions and its power-conjugated flow approximation basis (compatible with the exact derivation condition) to propose a new spectral symplectic reduction scheme. This choice will of course result in an accurate approximation of the homogeneous problem eigenfunctions. It will also give rise to accurate approximations for the solution in the case of non uniform resistivity and distributed control actions where the eigenfunctions of this problem are not Bessel functions anymore. Since the approximation bases are no longer eigenfunctions in the non uniform case, the method is then called a Galerkin scheme (rather than a spectral method). Since this spatial discretization scheme preserves the power product form, we call it symplectic (with respect to the power pairing). Finally, because the discretization scheme uses different bases for the effort and flow spaces, we call it mixed. We will now derive this mixed symplectic Galerkin scheme.

\subsection{Choice of the conjugated bases of eigenfunctions}

Computing the eigenfunctions of the operator appearing in the right hand side of the resistive diffusion equation (2.10) - in the constant and uniform resistivity case - is equivalent to solve Bessel equation (see Appendix A). Eigenvalues $\lambda_{k}$ may be computed from the zeros $s_{k}, k=$ $1 \ldots N-1$ of the first kind of Bessel functions of order $0, J_{0}$ and eigenfunctions may be written in terms of first kind of Bessel functions of order $1, J_{1}$ as:

$$
w_{k}^{f}(x)=J_{1}\left(\lambda_{k} x\right), \text { with } \lambda_{k}=\sqrt{s_{k} \frac{\eta}{\mu}}
$$

Therefore we will choose for the effort base functions:

$$
w_{i}^{e}(x)= \begin{cases}-\frac{1}{\lambda_{i}} J_{0}\left(\lambda_{i} x\right), & i=\overline{1 . . N-1} \\ 1 & i=N\end{cases}
$$

in such a way that the exact differentiation (compatibility) condition is satisfied, that is in this case:

$$
\begin{cases}\partial_{x} w_{k}^{e}(x) & =w_{k}^{f}(x), \forall k=\overline{1 . . N-1} \\ \partial_{x} w_{N}^{e}(x) & =0\end{cases}
$$

With this choice the derivation matrix $D$ in equation $\mathbf{f}=$ De reduces to to $\left[\mathbb{I}_{N-1} \mid 0\right] \in \mathcal{M}_{(N-1) \times N}$ where $\mathbb{I}_{N-1}$ is the identity matrix of order $N-1$, since

$$
\begin{array}{rlcc}
f & = & \partial_{x} e \\
\Rightarrow \quad \sum_{k=1}^{N-1} w_{k}^{f}(x) \mathbf{f}_{k} & = & \sum_{i=1}^{N}\left[\partial_{x} w_{i}^{e}(x)\right](\mathbf{e})_{i} \\
& = & \sum_{i=1}^{N-1} w_{i}^{f}(x)(\mathbf{e})_{i} \\
\Rightarrow & \quad \mathbf{f} & = & {\left[\mathbb{I}_{N-1} \mid 0\right] \mathbf{e}}
\end{array}
$$

\subsection{Projections of the constitutive equations}

The integration matrix, used in the power pairing (3.9) and needed to get the finite-dimensional representation of the Dirac interconnection structure, have to be computed. Elements in the diagonal and in the last row in this integration matrix may be computed exactly:

$$
\begin{aligned}
& M_{N k}=\int_{0}^{1} w_{k}^{f}(x) \mathrm{d} x \\
& =\left.\quad w_{k}^{e}(x)\right|_{0} ^{1} \quad=\frac{1}{\lambda_{k}} \\
& M_{k k}=\int_{0}^{1} w_{k}^{e}(x) \partial_{x} w_{k}^{e}(x) \mathrm{d} x \\
& =\left.\frac{1}{2}\left(w_{k}^{e}(x)\right)^{2}\right|_{0} ^{1} \quad=-\frac{1}{2}\left(\frac{1}{\lambda_{k}}\right)^{2}
\end{aligned}
$$

For the other elements (when $j \neq i$, hereafter) the Gauss quadrature formula with Chebyshev points will be used:

$$
\begin{array}{rlc}
M_{i j} & = & \int_{0}^{1} f_{i j}(x) \mathrm{d} x \\
& = & \frac{1}{2} \int_{-1}^{1} f(x) \mathrm{d}(1-2 x) \\
& =\frac{1}{2} \frac{\pi}{n} \sum_{k=1}^{m} \sqrt{1-\left(1-2 x_{k}\right)^{2}} f\left(x_{k}\right)
\end{array}
$$

where $f(x)=w_{i}^{e}(x) w_{k}^{f}(x)$ and $x_{k}=\frac{1-\cos \left(\frac{(2 k-1) \pi}{2(m-1)}\right)}{2}$. Indeed, unlike in the polynomial case, it is not possible to perform exact integration for a product of Bessel functions of different orders. Chebyshev quadrature points have been chosen to get a finite dimensional PCH model which may be easily compared to the previous ones (obtained from FD or symplectic collocation at the same points). The quadrature formula (4.6) is also used to determine the matrices $G$ and $R$ used in the finite dimensional storage and dissipation constitutive equations:

$$
\begin{aligned}
G_{i j} & =\int_{0}^{1} \frac{C_{2}}{\mu} w_{i}^{f}(x) w_{j}^{f}(x) \\
R_{i j} & =\int_{0}^{1} \frac{\eta}{C_{3}} w_{i}^{f}(x) w_{j}^{f}(x)
\end{aligned}
$$

This previous choice of conjugated bases functions obviously guarantees accurate eigenfunctions approximation for the problem with homogeneous boundary conditions and uniform resistivity since the approximation functions are the eigenfunctions. As it may be expected, it gives also still more accurate results for the eigenvalues approximations as illustrated in table 1 for the case $\eta=5.10^{-7}$.

\section{Numerical results}

\subsection{Non uniform resistivity}

In the previous section, when defining the approximation bases as spanned by the eigenfunctions of a simplified resistive diffusion equation, we neglected the dependence of the electrical resistivity $\eta$ with the time $t$ and the spatial radial coordinate $x$. However in real tokamaks, this 


\begin{tabular}{c|ccc}
$\begin{array}{c}\text { Theoretical } \\
\text { eigenvalues }\end{array}$ & \multicolumn{3}{|c}{ Numerical eigenvalues } \\
\hline-2.301056852 & -2.305253100 & -2.301848126 & -2.301466232 \\
-12.12413006 & -12.25659915 & -12.14481967 & -12.13359575 \\
-29.79659326 & -30.92406956 & -29.93017370 & -29.85455494 \\
-55.32237139 & -81.24868031 & -55.84563052 & -55.53473267 \\
-88.70194524 & & -90.35712887 & -89.30413565 \\
-129.9354296 & & -135.0263099 & -131.4267729 \\
-179.0228628 & & -297.6242993 & -182.5230680 \\
-235.9642604 & & & -244.5957644 \\
-300.7596298 & & & -530.5090590
\end{tabular}

Table 1: Eigenvalues with Bessel basis approximation functions for the case $\eta=5.10^{-7}$

resistivity is a function of the plasma state (noticeably of the plasma electronic temperature $T_{e}(x, t)$ and density $n_{e}(x, t)$, see [33] for details). Rather than including a multi-physics plasma model including the energy and mass material balance equations we will rather make use of an empirical expression for $\eta(t, x)$ (from [27]) which has been experimentally fitted for the Tore Supra tokamak discharge TS\#47673.

In this case, the chosen Bessel basis functions are no longer the eigenfunctions of the system. In fact, the theoretical eigenfunctions and eigenvalues are no more analytically solvable. The discretization method will be then called a symplectic Galerkin scheme since both the projection of the resistive diffusion equation and the cancellation of the corresponding residual are performed in the same approximation space generated by the Bessel functions.

The dissipation matrix $R$ now has to be computed online due to the time variations of the resistivity $\eta(t, x)$. In fact, the resistivity values supplied from the experimental data may be used to set the values of $\eta_{k}(t)$ of the resistivity at the discrete quadrature points $x_{k}$ used in the finite dimensional model. The dissipation $R$ matrix is computed by the online Gauss quadrature formula (4.6) according to:

$$
\begin{gathered}
R_{i j}(t)=\int_{0}^{1} \eta(t, x) \frac{R_{0}}{x} w_{i}^{f}(x) w_{j}^{f}(x) d x \\
=\frac{\pi}{2 n} \sum_{k=1}^{n} \eta\left(t, x_{k}\right) \frac{R_{0}}{x_{k}} w_{i}^{f}\left(x_{k}\right) w_{j}^{f}\left(x_{k}\right) \sqrt{1-\left(2 x_{k}-1\right)^{2}}
\end{gathered}
$$

The eigenvalues of the corresponding finite dimensional PCH model obtained with the proposed symplectic Galerkin method may now only be computed numerically. However one may check that the finite dimensional model still has purely dissipative eigenvalues (as expected from a diffusion equation!) and that these ones converge when $N$ increased. The results are in table 2 . where the eigenvalues are computed at time $t=11 s$ for the Tore Supra discharge TS\#47673.

\subsection{Test case definition}

Accuracy of the time response for the selected scenario (Tore Supra discharge TS\#47673, where plasma current

\begin{tabular}{cccc}
\multicolumn{4}{c}{ Numerical eigenvalues } \\
$\mathrm{N}=5$ & $\mathrm{~N}=8$ & $\mathrm{~N}=10$ & $\mathrm{~N}=20$ \\
\hline-0.316349428 & -0.315983205 & -0.315877522 & -0.315856805 \\
-2.673789607 & -2.372985846 & -2.372985846 & -2.371577391 \\
-14.74424065 & -6.357688599 & -6.278320313 & -6.267211833 \\
-359.7443273 & -14.08116991 & -12.01718458 & -11.87022206 \\
& -36.20739521 & -21.66293021 & -19.46289366 \\
& -129.9270811 & -43.38740206 & -30.44782740 \\
& -1724.357267 & -102.0884225 & -50.82178655 \\
& & -309.9311502 & -84.68730014 \\
& & -3355.769434 & -138.8389938
\end{tabular}

Table 2: Eigenvalues with Bessel base-functions and experimental $\eta(x, t=11 s) \in\left[2.10^{-8}, 2.10^{-6}\right]$, discharge TS\#47673

steps are performed) may not be evaluated only by simulation since experimental values have been used for the plasma resistivity. Therefore, in this section, we wish to present a validation of the global port Hamiltonian modeling and symplectic reduction approach, by comparing the simulation results against experimental data. The effort base functions size is chosen equal to $N=5, m=10$ Chebyshev points are used in the Gauss quadrature formula (4.6). The system input is the boundary condition $V_{\text {loop }}$ and the output is the plasma current $I_{p}$ determined as:

$$
I_{p}=-\left.\frac{x}{\mu R_{0}} \frac{\partial \psi}{\partial x}\right|_{x=1}=\left.\frac{x B_{\theta}}{\mu R_{0}}\right|_{x=1}=\frac{1}{\mu R_{0}} \sum_{k=1}^{N-1} w_{k}^{f}(1) \mathbf{b}_{k}
$$

The safety factor $q$-profile, which yields the ratio of the number of toroidal and poloidal field line turns (cf. [32]), is really important for MHD-stability and recent works on advanced tokamak control are based on its control, such as $[1,12]$. In first approximation, the $q$-profile is given by the expression presented in [5]:

$$
q=-\frac{1}{2 \pi} \frac{\partial \Phi}{\partial \psi}=-\frac{1}{2 \pi} \frac{\partial_{x} \Phi}{\partial_{x} \psi}
$$

$\Phi$ is the toroidal magnetic flux equal to $\partial_{x} \Phi=B_{\phi 0} a^{2} x$. $B_{\phi 0}$ denotes the constant toroidal magnetic field at the center of the plasma $(x=0)$.

In the test case, the non-inductive current source $j_{n i}$ is equal to the bootstrap current $j_{b s}$ (external non-inductive sources are set to zero), created by the plasma flux itself due to the charged particles bouncing back and forth along their banana orbit. This current source and the plasma resistivity are computed using experimental data and expressions based on the work of [27].

Furthermore, in a tokamak, the loop voltage $V_{\text {loop }}$ can't be set directly but is generated using central solenoid coil voltage. Thus on Tore Supra, a simple proportional controller is implemented between the plasma current and the coil voltage. On the proposed model, this controller is replaced by a proportional controller between $I_{p}$ and $V_{\text {loop }}$. The gain of the controller is tuned to get the same steady state error as on the real plant, cf. figures 2.a and 2.c. 


\subsection{Results}

The figure 2 shows the simulation results compared with experimental data. The coherence between our model and the experimental facility leads to the comparability in the result of Input-Output signals. The simulated loop voltage $V_{\text {loop }}$ is very closed to the real one, except on the begin of the pulse during the ramp-up phase when the shape of the plasma is not stable and so the minor and major radius of the plasma are time-dependent. This dependance is not taken into account in the port-Hamiltonian model and leads to the small observed difference in figure 2 .b before $t<3 \mathrm{~s}$. A zoom of the plasma current $I_{p}$ and loop voltage $V_{\text {loop }}$ presents the step response of the system in figure 2.c. A small delay appears between the experimental plasma current and the simulated one. This delay is coming from the actuator simplification done on the model: that the central solenoid dynamics are not modeled explains why the step response of the model is faster than the one of the real plant. a)

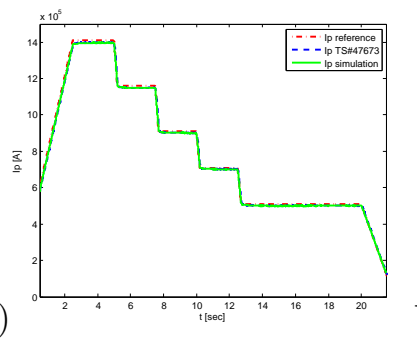

b)
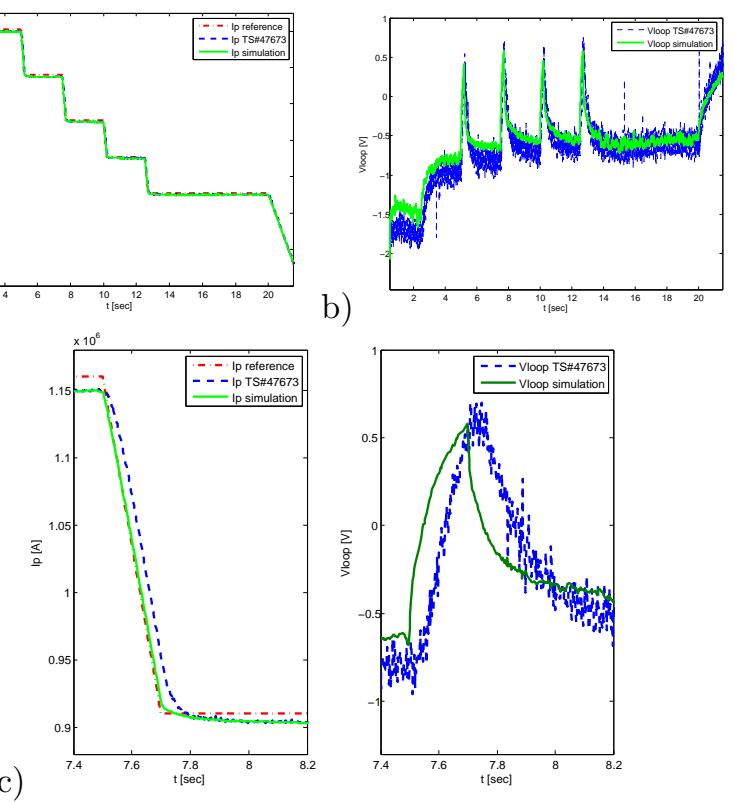

Figure 2: In-Output comparison

a) Plasma current $I_{p}$; b) Loop voltage $V_{\text {loop }}$;

c) $\left(I_{p}, V_{\text {loop }}\right)$ zoom at $t \in[7.4 s, 8.2 s]$

In figure 3 , the $q$-profiles are given at some sampling times and compared with the ones provided by the METIS code, a tokamak simulator developed for plasma scenario design and analysis (cf. [2]) in CEA Cadarache. This METIS code is a (complex) simulation code which uses the finite element method together with 'scale laws' and empirical formulas for constitutive physical closure equations.

From the previous comparisons, we may conclude that the finite dimensional model obtained with the proposed symplectic spatial discretization scheme may be used satisfactorily as a control model, at least for current density (or security factor) profiles regulation problems. It is a low order, with a low condition number and any usual explicit time integration scheme may be used. The simulation in the paper have been performed using ode45 solver from Matlab.
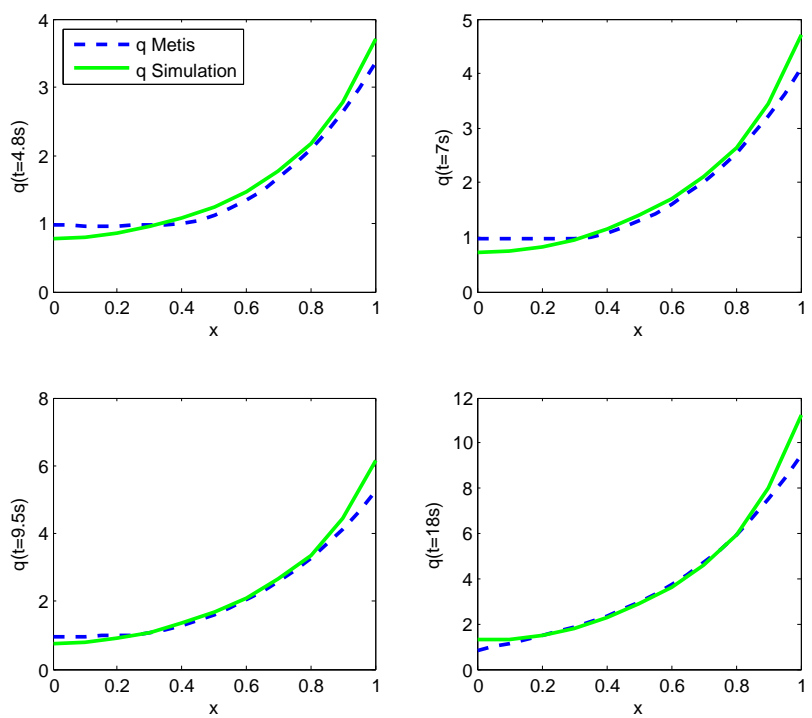

Figure 3: Safety factor $q$ comparison

\section{Conclusion}

In this paper we have suggested how classical pseudospectral methods for the spatial discretization of distributed parameters systems could be adapted in order to build structure preserving reduction schemes for a class systems of balance equations with boundary and/or distributed source terms. These balance equations have been represented using Stokes-Dirac interconnection structures. The spatial discretization schemes presented in this paper are then designed to preserve this structure. The basic idea to preserve the Stokes-Dirac interconnection structure is to choose different approximation bases for the thermodynamical variables, according to their geometric nature (the degree of the differential forms used to represent them). Then it becomes possible to get enough degrees of freedom and use them to make these bases compatible with the Stokes-Dirac underlying power pairing. Doing so, both the exterior derivative and the boundary operator may be discretized exactly and consequently the 'mixed' schemes developed with the proposed methodology are symplectic (or geometric) while the methods used to generate these mixed schemes are not.

Another point of interest in the paper is the discussion about the selection of the approximation bases. This is a general problem for pseudo-spectral models. However in the case of 'mixed' schemes, where several compatible approximation bases have to be selected, it was not obvious that interesting choices could happen. We claim that choosing, for one of the conjugated approximation 
bases, the set of eigenfunctions of a simplified problem may be an interesting choice. To illustrate the approximation bases choice methodology, we selected the Galerkin scheme among the possible pseudo-spectral methods. Then we showed that the skew-symmetry of the Stokes-Dirac structure gives rise to a set of eigenfunctions (Bessel functions in our example) for which a conjugated compatible base can easily be found. Finally, in the resistive diffusion equation example, we showed that this approach may reduce numerical oscillations in the presence of non homogeneous initial conditions or sharp distributed control.

Our geometric discretization approach, based on the port Hamiltonian formulation for open distributed parameters systems, has been used to generate a finite-dimensional approximation for a 1D control model for plasma dynamics in tokamaks. This confirmed the effectiveness of the approach in preserving some desired physical properties of the actual 1D model, namely spectral (eigenvalues and eigenfunctions) and energetic (conservativeness and passivity) properties. Moreover, although large simplification assumptions are made on the plasma geometry and properties to obtain the resistive diffusion equation, simulation results show good agreement with the experimental data and exhibit accuracy and qualitative behavior similar to the ones obtained from the complex reference code (METIS) for such plasma simulations.

Time discretization schemes for the obtained finite dimensional port-Hamiltonian models could be one of the next steps of this work. Indeed, discrete time control systems are used to operate tokamak devices and real time constraints still prevent the use of some non linear control laws. Structure preserving time discretization [14] would allow the development of passivity (or entropy) based control laws directly in discrete time.

\section{Appendix A. Theoretical eigenvalues for the resis- tive diffusion operator}

In this appendix we present the calculations needed to obtain eigenvalues and eigenfunctions of the simplified resistive diffusion equation (with homogeneous boundary condition and uniform resistivity). These eigenvalues are compared, in the core of the paper, with the numerical ones in order to prove the symplecticity of the proposed discretization schemes and their "spectral" accuracy. The corresponding eigenfunctions are used in the paper to generate conjugate compatible bases for the developed symplectic Galerkin method.

From the reduced sub-system in (2.12), the diffusion model is figured out as:

$$
\left(\begin{array}{c}
f_{e l \phi} \\
f_{m g \theta}
\end{array}\right)=\left(\begin{array}{cc}
0 & -\frac{\partial}{\partial \rho} \\
-\frac{\partial}{\partial \rho} & 0
\end{array}\right)\left(\begin{array}{c}
e_{e l \phi} \\
e_{m g \theta}
\end{array}\right)+\left(\begin{array}{l}
1 \\
0
\end{array}\right) f_{d \phi}
$$

where $f_{m g \theta}=-\partial_{t}\left(R_{0} B_{\theta}\right)=-\partial_{t} \tilde{B}_{\theta}$. For the considered case $f_{\text {el } \phi}=0, j_{n i}=0$, using the constitutive relation
(2.15), we get:

$$
\begin{aligned}
\left(\begin{array}{c}
f_{d \phi} \\
-\frac{\partial}{\partial t} \tilde{B}_{\theta}
\end{array}\right) & =\left(\begin{array}{cc}
0 & \frac{\partial}{\partial \rho} \\
-\frac{\partial}{\partial \rho} & 0
\end{array}\right)\left(\begin{array}{c}
e_{e l \phi} \\
e_{m g \theta}
\end{array}\right) \\
& =\left(\begin{array}{cc}
0 & \frac{\partial}{\partial \rho} \\
-\frac{\partial}{\partial \rho} & 0
\end{array}\right)\left(\begin{array}{cc}
\eta \frac{R_{0}}{\rho} & 0 \\
0 & \frac{1}{\mu} \frac{\rho}{R_{0}}
\end{array}\right)\left(\begin{array}{c}
f_{d \phi} \\
\tilde{B}_{\theta}
\end{array}\right)
\end{aligned}
$$

The eigenvalues $-s$ of the resistive diffusion equation are those complex values such that the second order equation

$$
-s \tilde{B}_{\theta}=\frac{1}{a^{2}} \frac{\partial}{\partial x}\left(\frac{\eta}{x}\left(\frac{\partial \frac{1}{\mu} x \tilde{B}_{\theta}}{\partial x}\right)\right)=\mathcal{L} \tilde{B}_{\theta} \text { where } x:=\frac{\rho}{a}
$$

has a non trivial solution (see the boundary conditions hereafter). In the case of constant coefficients (uniform permittivity and resistivity), the differential operator $\mathcal{L}$ is the classical Laplace operator in toric coordinates:

$$
\frac{1}{a^{2}} \frac{\partial}{\partial x}\left(\frac{\eta}{x}\left(\frac{\partial \frac{1}{\mu} x}{\partial x}\right)\right)
$$

In this case, one can prove using Green formula (on a disc coordinate cross section of the tokamak) that $-s$ is real and negative. These eigenvalues are calculated hereafter. For the case $\eta$ and $\mu$ constant, equation (A.3) becomes:

$$
x^{2} \frac{\partial^{2}}{\partial x^{2}} \tilde{B}_{\theta}+x \frac{\partial}{\partial x} \tilde{B}_{\theta}+\tilde{B}_{\theta}\left(\frac{\mu s}{\eta} x^{2}-1\right)=0
$$

Let $z^{2}=\frac{\mu s}{\eta} x^{2}$ (where $s>0$ ). Then the equation has the standard form of a Bessel equation:

$$
z^{2} \frac{\partial^{2}}{\partial z^{2}} \beta+z \frac{\partial}{\partial z} \beta+\beta\left(z^{2}-1\right)=0
$$

whose general solution is:

$$
a_{0} J_{1}(z)+a_{1} Y_{1}(z)
$$

where $J_{1}(z)$ and $Y_{1}(z)$ are 1st order Bessel functions of the first and second kind. In particular, $J_{1}(z)$ can be written:

$$
\begin{aligned}
J_{1}(z) & =\frac{1}{\pi} \int_{0}^{\pi} \cos (\tau-z \sin \tau) d \tau \\
& =\frac{1}{2 \pi} \int_{-\pi}^{\pi} e^{-i(\tau-z \sin \tau)} d \tau \\
& =\sum_{m=0}^{\infty} \frac{(-1)^{m}}{2^{2 m+1} m !(m+1) !} z^{2 m+1}
\end{aligned}
$$

The boundary conditions help us to determine the coefficient $a_{0}$ and $a_{1}$. The condition at $x=0$ forces $a_{1}=0$ 
as $Y_{1}(z)$ is unbounded when $z \rightarrow 0$. The other boundary condition $\left.\partial_{x}(x \beta)\right|_{1}=0$ implies:

$$
\left.\frac{\partial\left(z J_{1}(z)\right)}{\partial z}\right|_{z=\sqrt{\frac{s \mu}{\eta}}}=\left.z J_{0}(z)\right|_{z=\sqrt{\frac{s \mu}{\eta}}}=0
$$

Since Bessel functions satisfy

$$
\frac{\partial\left(z J_{1}\right)}{\partial z}=z J_{0}
$$

the eigenvalues may be calculated explicitly from the roots of the Bessel function $J_{0}(z)$. These zeros are represented on figure A.4 hereafter.

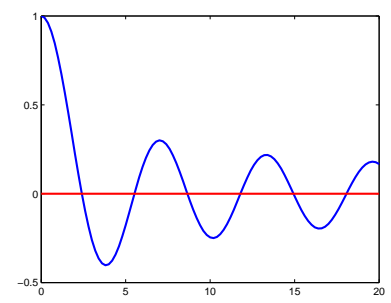

\begin{tabular}{|c|c|c|c|c|c|}
\hline$n$ & 1 & 2 & 3 & 4 & 5 \\
\hline \hline$z$ & 2.404825 & 5.520078 & 8.653727 & 11.791534 & 14.930917 \\
\hline
\end{tabular}

Figure A.4: The Bessel function $J_{0}(z)$ and its first zeros values

\section{Appendix B. A symplectic orthogonal collocation method for the spatial discretization of the resistive diffusion equation}

We develop in this appendix the symplectic collocation scheme generated with the proposed method applied to polynomial approximation bases for the effort and flow spatial profiles. These polynomial bases will be built from We analyze the accuracy of the resulting spectrum approximation and show that numerical oscillations occur when a distributed perturbation or sharp initial profiles are considered.

Let's $\xi_{j}(j=1 . . N-1)$ be the $N-1$ chosen collocation points for the flows and $\zeta_{i}(i=1 . . N)$ the $N$ collocation points chosen for the efforts. In the sequel to improve the order of the interpolation formulas and minimize the oscillations of the interpolation errors, zeros of respectively $(N-1)^{t h}$ and $N^{t h}$ orders Chebyshev polynomials will be chosen. Both notations $\mathbf{f} \in \mathbb{R}^{N-1}$ and $\mathbf{e} \in \mathbb{R}^{N}$ will be used to denote respectively the coordinates' vectors of the approximated flows and efforts in the corresponding approximation spaces. with the chosen orthogonal collocation method, the approximation basis for the flows is made with Lagrange interpolation polynomials at the collocation points $\xi_{j}(j=1 . . N-1)$ which satisfy the interpolation conditions

$$
w_{k}^{f}\left(\xi_{j}\right)= \begin{cases}1 & \text { if } j=k \\ 0 & \text { else }\end{cases}
$$

Most often, it is necessary to include boundary conditions in the approximation bases definitions to get satisfying results for the spectrum approximation. In the plasma resistive diffusion example, the boundary condition states that there is no flows source term at the center $x=0$ of the tokamak. The efforts at $x=0$ however may be non zero (and in fact usually is). Therefore we will consider the following additional conditions on the flow and effort bases:

$$
\begin{gathered}
w_{k}^{f}(0)=0 \\
\left.\frac{\partial w_{i}^{e}(x)}{\partial x}\right|_{x=0}=0 \\
w_{k}^{e}(0) \neq 0
\end{gathered}
$$

These conditions may be fulfilled with the choice of effort and flow approximations bases presented hereafter. Let $l_{i}^{N-1}$ denotes the $(n-1)^{t h}$ order Lagrange interpolation polynomial defined with:

$$
l_{i}^{N-1}(x):=\prod_{j=1, j \neq i}^{N} \frac{x-\zeta_{j}}{\zeta_{i}-\zeta_{j}}
$$

on the effort values at the boundary $x=0$ are satisfied by the functions

$$
w_{i}^{e}(x)=1-x^{2} \zeta_{i}^{2} l_{i}^{N-1}
$$

which may be used to generate the effort approximation space. The components of the derivation matrix are then:

$$
D_{j i}=\left.\frac{\partial}{\partial x}\left(w_{i}^{e}\right)\right|_{\xi_{j}}=\left.\left(-2 x \zeta_{i}^{2} l_{i}^{N-1}-x^{2} \zeta_{i}^{2} \frac{\partial}{\partial x}\left(l_{i}^{N-1}\right)\right)\right|_{\xi_{j}}
$$

Using this derivation matrix, a basis function for the flows which satisfies the exact derivation condition in (3.2) is:

$$
w^{f}(x)=\left(\frac{\partial}{\partial x} w^{e}(x)\right) D^{+}
$$

where $D^{+}$is the pseudo-inverse of $D$.

As $w^{e}(x)$ and $w^{f}(x)$ are both polynomial bases, the integral matrix $M$

$$
M_{i k}=\int_{0}^{1} w_{i}^{e}(x) w_{k}^{f}(x) d x
$$

may be easily exactly evaluated. Therefore, the projections of the storage and dissipation relations which results in the matrices $G$ (see subsection 3.4.1) and $R$ (see subsection 3.4.2) whose elements are:

$$
\begin{aligned}
G_{i j} & =\int_{0}^{1} \frac{1}{\mu} \frac{x}{R_{0}} w_{i}^{f}(x) w_{j}^{f}(x) \\
R_{i j} & =\int_{0}^{1} \eta \frac{R_{0}}{x} w_{i}^{f}(x) w_{j}^{f}(x)
\end{aligned}
$$

may be as well explicitly computed. The complete inputoutput model (3.31) has been computed for the case with 
uniform resistivity $\eta$ and homogeneous boundary conditions (both at $x=0$ and $x=1$ ). Then, eigenvalues of this model have been computed and compared with the theoretical ones (eigenvalues of the resistive diffusion operator with homogeneous boundary conditions, see appendix Appendix A) and with the ones obtained with a finite difference formula (see appendix Appendix D). Results are shown on tables B.4 and B.3 hereafter. The symplectic orthogonal collocation method is, from this spectrum approximation point of view, clearly more accurate, as it was expected.

\begin{tabular}{l|ccc}
\multirow{2}{*}{$\begin{array}{l}\text { Theoretical } \\
\text { eigenvalues }\end{array}$} & \multicolumn{3}{|c}{ Numerical eigenvalues } \\
-2.301056852 & $\mathrm{~N}=5$ & $\mathrm{~N}=8$ & $\mathrm{~N}=10$ \\
\cline { 2 - 4 }-12.12413006 & $-3,965414997$ & $-3,885480574$ & $-3,860185392$ \\
-29.79659326 & $-19,18940482$ & $-18,95845463$ & $-19,00658467$ \\
-55.32237139 & $-40,14692865$ & $-38,34709614$ & $-39,98776146$ \\
-88.70194524 & $-53,82013956$ & $-58,53622826$ & $-61,19522543$ \\
-129.9354296 & & $-85,81642985$ & $-82,15362798$ \\
-179.0228628 & & $-120,7439326$ & $-112,9192769$ \\
-235.9642604 & & $-127,9196240$ & $-155,4380422$ \\
-300.7596298 & & & $-180,4468519$ \\
& & & $-215,9294060$
\end{tabular}

Table B.3: Eigenvalues using a finite difference scheme and Chebyshev discretization points and $\eta=5.10^{-7}$

\begin{tabular}{l|ccc}
$\begin{array}{l}\text { Theoretical } \\
\text { eigenvalues }\end{array}$ & $\mathrm{N}=5$ & $\mathrm{~N}=8$ & $\mathrm{~N}=10$ \\
\hline-2.301056852 & -2.350753323 & -2.310110425 & -2.304769649 \\
-12.12413006 & -12.63581394 & -12.22630390 & -12.23545753 \\
-29.79659326 & -33.62142541 & -30.15754539 & -30.39891094 \\
-55.32237139 & -227.3150319 & -56.03293217 & -56.51610868 \\
-88.70194524 & & -92.85175835 & -88.81543981 \\
-129.9354296 & & -159.9060543 & -127.9699944 \\
-179.0228628 & & -1170.918746 & -176.7315844 \\
-235.9642604 & & & -317.0613604 \\
-300.7596298 & & & -2547.963503
\end{tabular}

Table B.4: Eigenvalues using the symplectic orthogonal collocation scheme with Lagrangian polynomial base-functions, Chebyshev collocation points and $\eta=5.10^{-7}$

Only the first eigenvalues approximations are reported in table B.4. The accuracy of higher frequencies eigenvalues quickly deteriorates which is quite usual when approximating infinite dimensional transfer functions with finite-dimensional models. Hopefully, in most closed loop control applications, these high frequencies eigenvalues of the control model are filtered and do not play any significant role.

Let us now consider an open system with a scenario where stationary values are chosen for the loop voltage $V_{\text {loop }}$ control action and a non zero distributed source term $j_{n i}$ is considered. The obtained radial profiles of the magnetic field component $B_{\theta}$ are illustrated on figure B.5, left (simulation case without distributed source term $j_{n i}=0$ ) and B.5, right (with $j_{n i} \neq 0$ ) and compared with the profiles supplied by the Finite Different (FD) method. a)

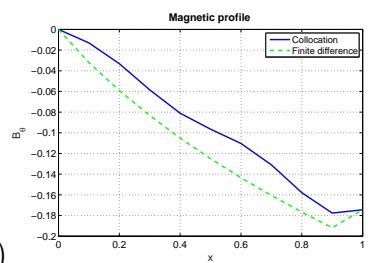

b)

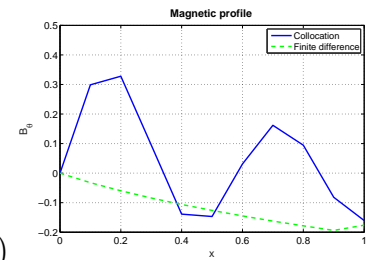

Figure B.5: $B_{\theta}$ profile with constant $\eta=5.10^{-7}$ controlled by $V_{l o o p}$ without $j_{n i}(\mathrm{a})$ and with $j_{n i}(\mathrm{~b})$

It may be observed that the magnetic profile $B_{\theta}$ has a saw-teeth (figure B.5, right) in the case of a non zero distributed control action, when using the symplectic collocation scheme. In fact, this is also the case - for the first time steps - when a sharp initial profile is chosen for the state variables. These numerical oscillations are not found in FD scheme. As it is shown in appendix Appendix C, these numerical oscillations come from a poor approximation of the eigenfunctions in the chosen Lagrange polynomials approximation bases (see figure C.6). These large oscillations results then from the boundary conditions (B.2) imposed to these "rigid" Lagrange polynomials in equation (B.4). Indeed, these oscillations disappear when classical Lagrangian polynomials are used rather than those in (B.4). But in this case, the problem boundary conditions are no longer satisfied!

\section{Appendix C. Error analysis for the symplectic col- location method}

Appendix C.1. Influence of the location of collocation points

Various locations of the collocation points lead to different precisions in the algorithm. In the sequel we compute the eigenvalues obtained with uniform, Legendre and Chebyshev collocation points. Thanks to the comparison table C.5, we may observe that the Chebyshev points leads to more accurate results than the two others choices. Furthermore, the choice of the effort collocation points reveals to have more influence than the one for the flux. This seems quite logical since the whole computations are based on the effort basis functions $w_{i}^{e}(x),(i=1 . . N)$.

The location of the collocation point does affect the precision but is not the explanation for the unwanted numerical oscillations in the reduced model. Therefore, we investigate hereafter another possible cause which is related to the basis functions choice itself.

\section{Appendix C.2. Influence of the basis functions choice}

We try now to indicate the approximation precision in eigenfunctions aspect. The error computation is referred to [25]. The theoretical eigenfunctions are calculated from the Bessel function of order $1 J_{1}$ like in equation (4.1):

$$
y_{k}(x)=J_{1}\left(\lambda_{k} x\right)
$$




\begin{tabular}{l|ccc} 
Theoretical & \multicolumn{3}{|c}{ Numerical eigenvalues } \\
eigenvalues & Uniform points & Legendre points & Chebyshev points \\
\hline-2.301056852 & $-2,4122190$ & $-2,3034030$ & -2.304769649 \\
-12.12413006 & $-18,6087633$ & $-11,8201217$ & -12.23545753 \\
-29.79659326 & $-44,4229304$ & $-27,9562188$ & -30.39891094 \\
-55.32237139 & $-92,0432864$ & $-52,8780494$ & -56.51610868 \\
-88.70194524 & $-160,4467269$ & $-86,8450446$ & -88.81543981 \\
-129.9354296 & $-323,5578853$ & $-131,9484170$ & -127.9699944 \\
-179.0228628 & 354,1681743 & $-208,9253302$ & -176.7315844 \\
-235.9642604 & $-627,2508525$ & $-330,3620033$ & -317.0613604 \\
-300.7596298 & $-2863,2871125$ & $-2453,3789667$ & -2547.963503
\end{tabular}

Table C.5: Comparison the precision of the different collocation point choice amount the points uniform, Legendre, Chebyshev $\eta=5.10^{-7}$

where $\lambda_{k}=\sqrt{s_{k} \frac{\eta}{\mu}}, k=1, \ldots N-1$. The approximating function is indicated by the Lagrange method:

$$
\hat{y}_{k}(x)=\sum_{j=1}^{N-1} y_{k}\left(\xi_{j}\right) w_{j}^{f}(x)
$$

with $\xi_{j}, j=1 \ldots N-1$ are the flux collocation points, $w_{j}^{f}(x)$ are the flux base functions.Approximating error is:

$$
E_{k}(x)=y_{k}(x)-\hat{y}_{k}(x)
$$

Then $E_{k}\left(\xi_{j}\right)=0, \forall j$ if $w^{f}$ are orthogonal function:

$$
w_{k}^{f}\left(\xi_{j}\right)= \begin{cases}1 & \text { if } j=k \\ 0 & \text { else }\end{cases}
$$

Appendix C.2.1. Symplectic collocation with boundary constraints

The chosen bases functions case are presented in Appendix B:

$$
w_{i}^{e}=1-x^{2} \zeta_{i}^{2} l_{i}, \quad l_{i}=\prod_{j=1 \neq i}^{N} \frac{x-\zeta_{j}}{\zeta_{i}-\zeta_{j}}
$$

where $\zeta_{i}, i=1 . . N$ are the collocation points for efforts, then $\operatorname{deg}\left(w^{e}\right)=N+1$.

The flux base functions are indicated from the effort ones as:

$$
w^{f}=\left(\partial_{x} w^{e}\right) D^{+}
$$

with pseudo inverse $D^{+}=D^{\prime}\left(D D^{\prime}\right)^{-1}$, then $\operatorname{deg}(\hat{y})=\operatorname{deg}\left(w^{f}\right)$ $=N$, and remind to the orthogonality of flux base functions $w_{j}^{f}(0)=0, \forall j$.

Let's take $N$ degree polynomials $p(x)=x \prod_{j=1}^{N-1}\left(x-\xi_{j}\right)$ , so $p(x)$ vanishes at 0 and $N-1$ flux collocation points $\xi_{j}: p\left(0, \xi_{j}\right)=0$. Then define a function $F(z)$ which is:

$$
F(z)=y(z)-\hat{y}_{k}(z)-\left[y_{k}(x)-\hat{y}_{k}(x)\right] \frac{p(z)}{p(x)}
$$

We have $\left\{0, \xi_{j}\right\}$ and $x$ are $N+1$ roots of $F(z)$. It's possible thus to apply $N$ times the Rolle's theorem on $F(z)$, we get:

$$
F(z)^{(N)}=y(z)^{N}-\hat{y}(z)^{N}-[y(x)-\hat{y}(x)] \frac{N !}{p(x)}
$$

then there's at least a $\zeta \in\left[0, \xi_{N-1}\right]$ satisfies $F(\zeta)=0$. In orders words:

$$
E(x)=y(x)-\hat{y}_{k}(x)=\left[y(\zeta)^{N}-\hat{y}(\zeta)^{N}\right] \frac{p(x)}{N !}
$$

However, as $\operatorname{deg}(\hat{y})=N$ the constant $y(\zeta)^{N}-\hat{y}(\zeta)^{N}$ also has an important effect on $E(x)$.The figure C.6 shows the very significant error in eigenfunction approximation, this polynomials base function choice just sticks at the collocation points, such as with the first and the fifth eigenvalues.

a)

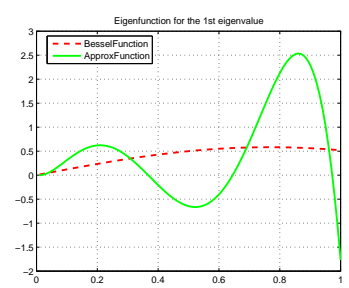

Figure C.6: The first (a) and fifth (b) calculated eigenfunctions with boundary conditions $w_{i}^{e}=1-x^{2} \zeta_{i}^{2} l_{i}$ vs theoretical Bessel eigenfunctions

Appendix C.2.2. Symplectic collocation without boundary constraints

Once realizing that the constant error left in the previous base function choice, consider now the case without boundary conditions, we simply take effort base function equal to the Lagrange polynomials of degree $N-1$, $\operatorname{deg}\left(w^{e}\right)=N-1$ :

$$
w_{i}^{e}=l_{i}, \quad l_{i}=\prod_{j=1 \neq i}^{N} \frac{x-\zeta_{j}}{\zeta_{i}-\zeta_{j}}
$$

Similar to the previous case, flux base functions $w^{f}$ has the degree of $N-2$ :

$$
\operatorname{deg}(\hat{y})=\operatorname{deg}\left(w^{f}\right)=N-2
$$

This time the polynomials $p(x)$ is taken as:

$$
p(x)=\prod_{j=1}^{N-1}\left(x-\xi_{j}\right)
$$

where $\operatorname{deg}(p)=N-1$ and of course $p\left(\xi_{j}\right)=0$.

With the same function $F(z)$ as before:

$$
F(z)=y(z)-\hat{y}_{k}(z)-\left[y_{k}(x)-\hat{y}_{k}(x)\right] \frac{p(z)}{p(x)}
$$


We have now $N$ roots $\left(\xi_{i}\right.$ and $\left.x\right)$ of $F(z)$, then $N-1$ times the Rolle's theorem is applied:

$$
F(z)^{N-1}=y(z)^{N-1}-\hat{y}(z)^{N-1}-[y(x)-\hat{y}(x)] \frac{(N-1) !}{p(x)}
$$

then there's at least a $\zeta \in\left[\xi_{1}, \xi_{N-1}\right]$ satisfies $F(\zeta)=0$. In orders words

$$
E(x)=y(x)-\hat{y}_{k}(x)=\left[y(\zeta)^{N-1}-\hat{y}(\zeta)^{N-1}\right] \frac{p(x)}{(N-1) !}
$$

In this case $\hat{y}(z)^{N-1}=0$ since $\operatorname{deg}(\hat{y})=N-2$, the approximation error is thus of order of $y^{N}=J_{1}^{N}$, and it has the order of $10^{-5}$ with $N=10$. In the figure C.7, the eigenfunction is better approached with the first eigenvalue, and has the same order with the fifth eigenvalue. a)

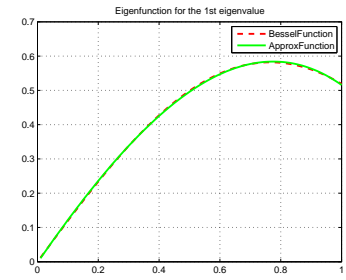

b)

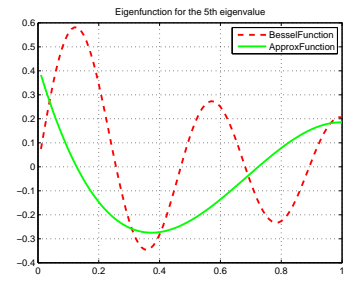

Figure C.7: Simulation for the case of $w e_{i}=l e_{i}$ with constant $\eta=5.10^{-7}$, without boundary conditions: a) $1^{\text {st }}$ eigenfunction approximation; b) $5^{\text {th }}$ eigenfunction approximation

It turns out that, although giving a very accurate eigenvalue approximation, this symplectic mixed orthogonal collocation discretization scheme poorly approximates the original (resistive diffusion) problem eigenfunctions. This gives rise to unwanted numerical oscillations in the simulation when a distributed source term or sharp initial profiles are considered.

\section{Appendix D. The finite difference scheme used as a reference}

In section 4, a finite-difference scheme is used for the spatial discretization of the resistive diffusion equation written in the port-Hamiltonian form (2.13). The accuracy of the spectrum approximation obtained with this scheme is then compared with the accuracy obtained with the proposed symplectic collocation and Galerkin schemes. To achieve a fair comparison, a very special case has to be considered. First, the grid points for the finite difference scheme are zeros of Chebyshev polynomials. Indeed, the finite difference scheme is not symplectic anymore (in the classical sense for closed Hamiltonian systems) for uniformly spaced grid points. Besides, the spectrum accuracy is maximal with Chebyshev points. Then, homogeneous boundary conditions have to be considered. Indeed, whatever the choice of discretization points is, the finite difference scheme is not symplectic anymore (with respect to the bilinear power product used to defined the StokesDirac structure) for open systems (with non autonomous boundary conditions). This will result in unstable and oscillating modes (and meaningless values for the spectrum) in the finite difference approximated model while the resistive diffusion model itself is of course purely dissipative. The finite difference scheme used to solve the resistive diffusion equation (for the unknown function $\tilde{B}_{\theta}=R_{0} B_{\theta}$ ) with these assumptions is presented hereafter. We start from the resistive diffusion equation:

$$
\begin{array}{rlc}
\frac{\partial \tilde{B}_{\theta}}{\partial t} & =\frac{\eta}{\mu a^{2}} \frac{\partial}{\partial x}\left(\frac{1}{x} \frac{\partial}{\partial x}\left(x \tilde{B}_{\theta}\right)\right)-\eta \frac{\partial}{\partial x} j_{n i} \\
& =\frac{\eta}{\mu a^{2}}\left(\frac{1}{x^{2}} \tilde{B}_{\theta}+\left(\frac{1}{x}+1\right) \frac{\partial}{\partial x} \tilde{B}_{\theta}+x \frac{\partial^{2}}{\partial x^{2}} \tilde{B}_{\theta}\right)-\eta \frac{\partial}{\partial x} j_{n i}
\end{array}
$$

By denoting $\mathbf{b}_{\mathbf{i}}, \quad(i=1 . . N)$ the approximated values of $\tilde{B}_{\theta}$ at the discretization points $x_{i}$ (zeros of the $(N-2)^{t h}$ order Chebyshev polynomial, completed with the two boundary values, in our case), one gets:

$$
\begin{aligned}
\frac{\partial \mathbf{b}_{i}}{\partial t}= & \frac{\eta}{\mu a^{2}}\left[-\frac{1}{x_{i}^{2}} \mathbf{b}_{i}+\left(\frac{1}{x_{i}}+1\right) \frac{\mathbf{b}_{i+1}-\mathbf{b}_{i-1}}{\delta_{i+1}+\delta_{i}}\right. \\
& \left.+2 x_{i} \frac{\mathbf{b}_{i+1}-2 \mathbf{b}_{i}+\mathbf{b}_{i-1}}{\delta_{i+1}^{2}+\delta_{i}^{2}}\right] \forall i=2 . .(N-1)
\end{aligned}
$$

where

$$
\left\{\begin{array}{ccc}
\delta_{i+1} & = & x_{i+1}-x_{i} \\
\delta_{i} & = & x_{i}-x_{i-1}
\end{array}\right.
$$

The boundary conditions give the missing values for $\mathbf{b}_{1}$ and $\mathbf{b}_{N}$ :

$$
\left\{\begin{array}{ccccc}
\left.\tilde{B}\right|_{x_{1}=0} & =0 & \Rightarrow & \mathbf{b}_{1}= & 0 \\
\left.\partial_{x}(x \tilde{B})\right|_{x_{N}=1} & =0 & \Rightarrow & \mathbf{b}_{N}= & \frac{\mathbf{b}_{N-1}}{2-x_{N-1}}
\end{array}\right.
$$

Written in matrix form (convenient for instance for eigenvalues computations) the diffusion equation becomes:

$$
\frac{\partial \mathbf{b}}{\partial t}=\frac{\eta}{\mu a^{2}} \underbrace{\left[\begin{array}{ccccc}
\beta_{2} & \gamma_{2} & & & \\
\ddots & \ddots & \ddots & & \\
& \alpha_{i} & \beta_{i} & \gamma_{i} & \ddots \\
& & \ddots & \ddots & \left(\beta_{N-1}+\frac{1}{2-x_{N-1}} \gamma_{N-1}\right)
\end{array}\right]}_{A} \mathbf{b}
$$

with $\mathbf{b}:=\left(\mathbf{b}_{2}, \ldots, \mathbf{b}_{N-1}\right)^{T}$ and where:

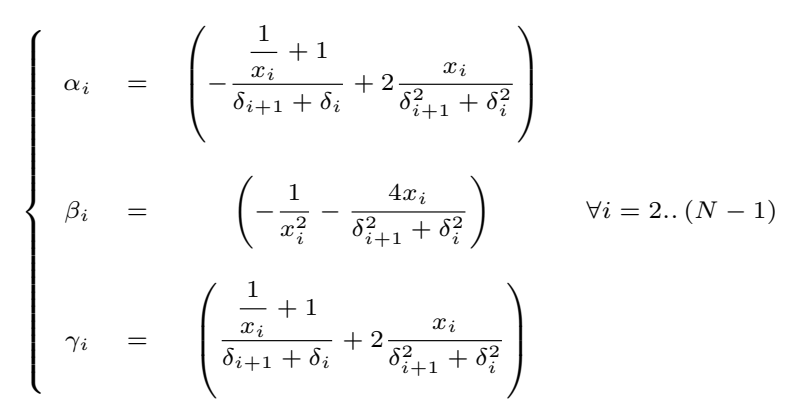

As it has been noticed, this finite difference scheme with over $N=200$ points has spectral properties (see table B.3) which are comparable to those obtained with the collocation method with about $N=10$ discretization points (see table B.4). Basically, the eigenvalues calculated from 
the characteristic matrix $A$ in the equation D.4 (which is however a tridiagonal matrix) converge to the theoretical values as $\frac{1}{N}$, while those obtained with the collocation method (and its full matrix) as $\frac{1}{N^{2}}$.

[1] F.B. Argomedo, C. Prieur, E. Witrant, and S. Brémond. A strict control lyapunov function for a diffusion equation with time-varying distributed coefficients. IEEE Transactions on Automatic Control (2012), 2012.

[2] J.F. Artaud, V. Basiuk, F. Imbeaux, M. Schneider, J. Garcia, G. Giruzzi, and et al. The cronos suite of codes for integrated tokamak modelling. Nuclear Fusion, 50(043001), 2010.

[3] A. Baaiu, F. Couenne, D. Eberard, C. Jallut, Y. Legorrec, L. Lefèvre, and B. Maschke. Port-based modelling of mass transfer phenomena. Mathematical and Computer Modelling of Dynamical Systems, 22(1):1-22, 2009.

[4] A. Baaiu, F. Couenne, Y. Legorrec, L. Lefèvre, and M. Tayakout-Fayolle. Structure-preserving infinite dimensional model reduction: Application to adsorption processes. Journal of Process Control, 19(3):394-404, 2009.

[5] J. Blum. Numerical Simulation and Optimal Control in Plasma Physics. Gauthier-Villars, 1989.

[6] A. Bossavit. Computational Electromagnetism. Academic Press, 1998.

[7] T.J. Courant. Dirac manifolds. Trans. American Math. Soc. 319, pages 631-661, 1990.

[8] M. Dalsmo and A.J. van der Schaft. On representations and integrability of mathematical structures in energy-conserving physical systems. SIAM Journal of Control and Optimization, 37(1):54-91, 1999.

[9] B.A. Finlayson. The method of weighted residuals and variational principles. Academic Press, 1972.

[10] B. Fornberg. A Practical Guide to Pseudospectral Methods. Cambridge University Press, 1996.

[11] A.A Franco, P. Schott, C. Jallut, and B.M. Maschke. A multiscale dynamic mechanistic model for transient analysis of pefcs. Fuel Cells: From Fundamentals to Systems, 7(2):99-117, April 2007.

[12] O. Gaye, E. Moulay, S. Brémond, L. Autrique, R. Nouailletas, and Y. Orlov. Sliding mode stabilization of the current profile in tokamak plasmas. Conference on Decision and Control (2011), Orlando, Florida, USA, December 2011.

[13] G. Golo, V. Talasila, A.J. van der Schaft, and B. Maschke. Hamiltonian discretization of boundary control systems. Automatica, 40(5):757-771, 2004.

[14] E. Hairer, C. Lubich, and G. Wanner. Geometric numerical integration : structure-preserving algorithms for ordinary differential equations, volume 31 of Springer Series in Computational Mathematics. Springer-Verlag, Berlin Heidelberg, 2002. ISBN 3-540-43003-2.

[15] B. Hamroun, L. Lefevre, and E. Mendes. Port-based modelling for open channel irrigation systems. Transactions on Fluid $\mathrm{Me}$ chanics, 1(12):995-1009, 2006.

[16] Y. Le Gorrec, H. Zwart, and B.M. Maschke. Dirac structures and boundary control systems associated with skew-symmetric differential operators. SIAM J. of Control and Optimization, 44(5):1864-1892, 2005.

[17] A. Macchelli and B.M. Maschke. Modeling and Control of Complex Physical Systems - The Port-Hamiltonian Approach, chapter Infinite-dimensional Port-Hamiltonian Systems, pages 211272. Springer, Sept. 2009. ISBN 978-3-642-03195-3.

[18] A. Macchelli and C. Melchiorri. Modeling and control of the Timoshenko beam. the Distributed Port Hamiltonian approach. SIAM Journal On Control and Optimization, 43(2):743-767, 2004.

[19] B. Maschke and A.J. van der Schaft. Advanced Topics in Control Systems Theory. Lecture Notes from FAP 2004. Lecture Notes on Control and Information Sciences. Springer, 2005 (115-154).

[20] B.M. Maschke and A. J. van der Schaft. Canonical interdomain coupling in distributed parameter systems: an extension of the symplectic gyrator. In Proc. Int. Mechanical Engineering Congress and Exposition, New- York, USA, Nov. 2001. ASME.

[21] P. Moreau, S. Brémond, D. Douai, A. Geraud, P.Hertout, M.Lennholm, D. Mazon, F. Saint-Laurent, and Tore Supra team. Plasma control in tore supra. Fusion science and technology, 56:1284-1299, July 2009.

[22] R. Moulla, L. Lefèvre, and B. Maschke. Pseudo-spectral methods for the spatial symplectic reduction of open systems of conservation laws. Journal of Computational Physics, 231(4):19721992, December 2012.

[23] G. Nishida, K. Takagi, and B. Maschke. Multiscale distributed port-hamiltonian representation of ionic polymer-metal composite (ipmc). In Proc. IFAC World Congress (2008) 23002305.

[24] P.J. Olver. Applications of Lie Groups to Differential Equations, volume 107 of Graduate texts in mathematics. Springer, New-York, ii edition, 1993.

[25] A. Ralston and P. Rabinowitz. A first course in numerical analysis. Dover Publication,Inc, Mineola, Newyork, 2nd edition, 2001.

[26] S. Reich. Multi-symplectic Runge-Kutta collocation methods for Hamiltonian wave equations. Journal of Computational Physics, 157:473-499, 2000.

[27] O. Sauter, C. Angioni, and Y.R. Lin-Liu. Neoclassical conductivity and bootstrap current formulas for general axisymmetric equilibria and arbitrary collisionality regime. Physic of Plasma, 6(7):2835-2839, July 1999.

[28] A.J. van der Schaft and B.M. Maschke. The Hamiltonian formulation of energy conserving physical systems with external ports. Archiv für Elektronik und Übertragungstechnik, 49(5/6): 362-371, 1995.

[29] A.J. van der Schaft and B.M. Maschke. Hamiltonian formulation of distributed parameter systems with boundary energy flow. Journal of Geometry and Physics, 42:166-174, 2002.

[30] N.M.T. Vu. The Port-Hamiltonian Approach for the Modelling, Reductionr and Control of Plasma Dynamics in Tokamaks. $\mathrm{PhD}$ thesis, Univ. Grenoble Alpes, Grenoble, France, November 2014.

[31] Trang.N.M. Vu, L. Lefèvre, R. Nouailletas, and S. Brémond. Structure preserving reduction for thermo-magneto plasma control model. The 21st International Symposium on Mathematical Theory of Networks and Systems (2014), Groningen, Netherlands, July 07-11 2014.

[32] J. Wesson. Tokamaks. Third edition. Oxford Science Publications, 2004.

[33] E. Witrant, E. Joffrin, S. Brémond, G. Giruzzi, D. Mazon, O. Barana, and P. Moreau. A control-oriented model of the current profile on tokamak plasma. Plasma Physics and Controlled Fusion, 49:1075-1105, 2007. 